Reçu le II mai 1922.

\title{
CONTRIBUTION A L'ÉTUDE DE LA PHYSIOLOGIE DU CERVELET
}

\section{LA FONCTION INHIBITRICE DU PALEO-CEREBELLUM}

\author{
PAR \\ Fréteric Bremer \\ (Laboratoire de recherches" chirurgicales, Harvard, et Laboratoire \\ de Physiologie, Oxford).
}

(20 figures)

\section{INTRODUCTION}

ES recherches de ces dernières années ont définitivement établi L la distinction entre les phénomènes toniques et cloniques (1) de l'activité des muscles striés. Ces modalités de la contractilité sont nettement séparées par leurs caractères physiques, chimiques et biologiques : la plasticité du muscle contracturé, sa dépense énergétique infime et d'un chimisme peut-être particulier (créatine), son infatigabilité, s'opposent à l'élasticité, à la dépense énergétique considérable et qui utilise les hydrocarbonés, à la fatigabilité rapide du muscle tétanisé. Les mécanismes nerveux de ces formes de l'activité musculaire sont probablement distincts aussi. La constatation [BUYTENDYK, (1), Einthoven (2)] d'ailleurs dans la contracture de décérébration, d'oscillations électriques, de faible amplitude, mais d'une fréquence analogue à celles que l'on observe dans la contraction volontaire, ne prouve pas que cette contracture et les autres phénomènes toniques soient un tétanos ayant la fréquence de ces oscillations : il faudrait d'abord prouver que le rythme électrique de la contraction volontaire est le rythme d'un tétanos. Or Forbes et Rappleye (3) ont

(1) Les auteurs anglais emploient de préférence l'épithète de phasique pour désigner la modalité de contractilité qui s'oppose à la contractilité tonique. Le manque d'un terme à la fois expressif et adéquat se fait sentir đans les deux langues: Les expressions sarcoplasmatique et myofibrillaire qui préjugent d'une question non résolue ne peuvent pas être employées. 
montré que la fréquence de ce rythme dépend complètement de conditions musculaires locales et qu'elle ne représente nullement la fréquence du rythme nerveux, fréquence qui est tout à fait inconnue. D'ailleurs ce rythme, de 70 oscillations par seconde environ (rythme de Piper) paraît être un phénomène musculaire très général : H. Schaeffer (4) l'a retrouvé dans la contracture de Tiegel, provoquée en faradisant des muscles complètement isolés de leurs connections centrales. Au surplus il est notoire que souvent les méthodes les plus sensibles ne peuvent déceler aucun phénomène électrique dans des muscles contracturés (MAGNus et LILJESTRAND (5), WeRTHEIM-SALOMONSON (6).

Sous le nom de phénomènes toniques, on ne comprend pas seulement des états musculaires statiques comme les contractures pathologiques ou les rigidités musculaires normales qui maintiennent des attitudes, mais des modifications en plus ou en moins de ce tonus de base accompagnent presque toutes les réactions musculaires de l'organisme et en sont la composante tonique. Elles peuvent même exister isolément : l'observation a révélé la nature essentiellement tonique de certains réflexes, par exemple, les réflexes vestibulaires toniques [MAGNus et De KLEYN (7)], les réactions obtenues par l'excitation des faisceaux longitudinaux postérieurs et des noyaux rouges [Graham Brown (8)], le réflexe de Babinski [WertheimSALOMONSON (6)].

Rappelons que le tonus musculaire, et les contractures qui en sont l'exagération, sont l'expression d'un réflexe permanent qui a son origine dans le muscle lui-même (réflexe proprioceptif) et qui nécessite l'intégrité de certains centres, d'autant plus nombreux et situés d'autant plus haut sur l'axe nerveux, que celui-ci est plus évolué : ainsi la destruction de l'écorce motrice rolandique chez l'homme a comme conséquence une forte hypotonie musculaire. Cette hypotonie est beaucoup moins marquée pour la même lésion chez le singe anthropoïde ; elle est inappréciable chez le chat. On appelle ces centres des "sources de tonus".

D'autre part, le tonus est constamment soumis à des influences inhibitrices, probablement aussi de nature réflexe et qui sont centralisées dans des noyaux gris : centres inhibiteurs ; on connait l'importance inhibitrice du globus pallidus et du locus niger. La destruction d'un centre inhibiteur important entraîne la contracture muscu- 
laire. Un état tonique déterminé est donc la résultante de deux forces opposées, l'une renforçatrice, l'autre inhibitrice. Le lieu de cet antagonisme n'est pas connu, mais il est difficile de concevoir qu'il se produise ailleurs que sur des neurones ou leurs synapses.

Mais il est d'autres réflexes proprioceptifs que ces grands réflexes permanents et silencieux du tonus et de son inhibition. Tout muscle, raccourci ou étiré, envoie des influx qui reviennent à leur point de départ après s'être réfléchis dans la moelle ou dans des centres encore mal précisés. Sur chaque contraction réflexe se greffe une seconde contraction, réflexe autogène, qui la renforce et la prolonge. C'est ce réflexe proprioceptit qui constitue la plus grande partie de l' "after discharge " des réflexes spinaux, cette persistance de la contraction après la cessation de l'excitation et qui fusionne les secousses cloniques d'un tétanos réflexe imparfait. D'autre part, un muscle strié, en état de tonus, garde, lorsqu'il est allongé, les longueurs qu'on lui impose, et sa tension reste la même malgré les changements de longueur. Cette plasticité n'est pas simplement l'expression d'un état physique de la substance contractile, elle est aussi le résultat d'une régulation délicate par des réflexes proprioceptifs. En raison du rôle qu'elle doit jouer dans le maintien des attitudes, on l'a qualifiée de "posturale".

Ces notions, dont nous devons la plupart aux belles recherches de SHERRINGTON et qu'il était nécessaire de rappeler brièvement, permettent de mieux concevoir l'utilité biologique du tonus musculaire : le tonus statique assure automatiquemant et avec une dépense énergétique très faible le maintien des attitudes, et entre autres de la plus importante de toutes, celle de la station debout : d'où l'importance du tonus des muscles extenseurs, c'est-à-dire de ceux qui contrebalancent constamment l'action de la pesanteur, d'où aussi le fait que ce tonus dépend étroitement des organes sensoriels, les labyrinthes qui règlent l'équilibre statique. De plus, le tonus intervenant comme une composante des mouvements volontaires et réflexes, en assure le fondu et l'harmonieuse souplesse en même temps qu'en les prolongeant et les renforçant, il diminue d'autant la dépense énergétique de l'organisme.

Le rôle du tonus musculaire étant ainsi défini, il n'est pas surprenant que les activités du cervelet se révèlent comme étant principalement des activités toniques. La statique normale est assurée par un 
mécanisme essentiellement tonique : or l'astasie n'est-elle pas l'un des symptômes cardinaux du déficit cérébelleux ? Les caractères de la motricité des cérébelleux, brusquerie, amplitude exagérée, tremblement, fatigabilité, ne sont-ils pas ceux de la contraction réflexe des muscles atoniques tels qu'ils ont été décrits par SHERRINGTON ? Pendant longtemps cependant, l'observation clinique parut en désaccord avec l'expérience physiologique en ce qui concerne l'importance de l'hypotonie musculaire dans les désordres cérébelleux. Cela était dû sans doute à ce que, en temps ordinaire, les syndrômes cérébelleux purs sont fort rares. Mais l'observation, au cours de la dernière guerre, de nombreux blessés du cervelet [GoRDon-HoLmes (9)] a montré chez eux la constance et l'intensité de l'hypotonie musculaire du côté atteint.

Cependant, le mécanisme de l'action du cervelet sur le tonus reste obscur. On pensait que le cervelet était essentiel à l'exécution des réflexes toniques labyrinthiques et cervicaux. DE KLEYN et Magnus (10) ont démontré qu'il n'en est rien. De plus, ces auteurs viennent de faire la même démonstration pour certains des réflexes qu'ils ont appelé réflexes de redressement, lesquels ont pour but le rétablissement actif de la station debout. Ceux de ces réflexes qui sont d'origine vestibulaire, s'exécutent encore après l'ablation complète du cervelet. Le cervelet n'est donc pas un centre vestibulaire supérieur comme on l'a cru longtemps ; il n'intervient pas dans l'exécution des réflexes élémentaires dont l'action synergique assure le rétablissement et le maintien de l'équilibre statique. D'ailleurs, l'analogie entre le syndrôme vestibulaire et le syndrôme cérébelleux n'est pas si étroite qu'on l'a pensé ; les mouvements de roulements n'appartiennent pas à la symptomatologie du cervelet et sont dûs à la lésion concomitante des noyaux vestibulaires [DE KLEYN et Magnus (10)].

D'autre part, la conception de localisations cérébelleuses dans le sens de BoLk (11) semble bien devoir être abandonnée. On sait que Bolk, se basant sur la comparaison des cervelets des mammifères, a localisé dans l'écorce cérébelleuse une série de centres réglant chacun le tonus de territoires musculaires déterminés. Son hypothèse fut soumise à un contrôle expérimental et les premières recherches lui paraissaient favorables, au moins en ce qui concerne la représentation des membres antérieurs et postérieurs dans les deux parties du lobe ansiforme. 
Mais Gordon Holmes (9) n'a pu se convaincre de l'existence de ces localisations chez l'homme et il pense que les résultats sur l'animal sont dûs à la lésion, difficile à éviter, des noyaux centraux. Un travail expérimental tout récent de Troell et Hosser (12) doit engager au même scepticisme, et d'ailleurs les recherches d'anatomie comparée de IngVAR (13) ont enlevé à l'hypothèse de Bolk sa base morphologique. Le cervelet n'est donc pas une mosaïque de centres toniques. S'il existe des localisations fonctionnelles dans l'écorce cérébelleuse, le principe doit en être autre et elles doivent avoir comme base la différenciation anatomique que révèle l'étude des connexions afférentes et efférentes de l'organe, ainsi que son développement phylogénétique et embryologique.

Dans le présent travail, je me suis proposé d'étudier les activités que manifeste le cervelet chez l'animal décérébré, la transsection du tronc cérébral étant faite immédiatement en avant des tubercules quadrijumeaux antérieurs et en arrière des tubercules mammillaires. La décérébration constitue par elle-même un moyen d'analyse, puisqu'elle isole le cervelet de ses associations avec le thalamus et l'écorce cérébrale. De plus, la méthode offre des avantages particuliers et l'on sait combien elle a déjà été utile à l'étude de la physiologie nerveuse : non nécessité de la narcose, présence d'un " tonic back-ground " qui amplifie les altérations expérimentales $\mathrm{du}$ tonus. On sait par ailleurs que la rigidité de décérébration l'est que l'exagération du tonus normal des extenseurs, libéré d'une inhibition. Comme le tonus normal, elle nécessite l'intégrité de l'arc spinal et de centres métencéphaliques dont les noyaux vestibulaires sont probablement les plus importants [THIELE (14)], bien que les influx labyrinthiques ne soient pas essentiels [SHERRINGToN (15)]. Les centres inhibiteurs que la décérébration met hors de cause sont probablement le globus pallidus et les noyaux sous-thalamiques (Thiele 14), parmi lesquels le locus niger est peut être le plus important (TRETIAKOFF et Bremer) (16). Il arrive fréquemment qu'au bout de quelques heures la contracture d'extension fait place à une contracture de flexion, ce qui fournit l'occasion d'étudier le tonus des fléchisseurs.

En prenant soin de faire la transsection du tronc cérébral suffisamment antérieure, on conserve les principales vcies efférentes du 
cervelet. D'autre part, il était légitime de supposer que le palaeocerebellum, dont les connexions afférentes restent intactes, exerce des fonctions indépendamment des centres sus-jacents.

On peut, semble-t-il, envisager l'activité du cervelet dans ses rapports avec la contracture d'extension de l'animal décérébré à un triple point de vue : le cervelet, comme source de tonus, comme centre inhibiteur du tenus, enfin comme centre producteur de réactions toniques posturales.

\section{Le rôle du cervelet et des noyaux rouges, dans le maintien de la rigidité de décérébration.}

Dès le début de ses recherches sur la rigidité, SHERRINGTon (15) fit la constatation assez inattendue que celle-ci peut persister après l'ablation complète du cervelet. THIELE (14) constata que, ni l'écorce cérébelleuse, ni les noyaux rouges n'interviennent dans le maintien de la contracture et que celle-ci n'est atteinte que par la lésion des noyaux vestibulaires. Horsley (17) pensait que la contracture de décérébration dépend de l'intégrité des noyaux cérébelleux et paracérébelleux. Plus tard, WEED (18) tenta de démontrer que le cervelet et les noyaux rouges jouent un rôle important dans le maintien de la rigidité. Mais De KLeYn et Magnus (10) apportèrent la preuve qu'une rigidité marquée apparaît régulièrement et persiste malgré l'ablation histologiquement complète du cervelet.

Cependant il ne résulte pas de ce fait que le cervelet ne lui fournit aucun appoint. Car s'il est vrai qu'il existe dans le vermis un mécanisme inhibiteur, la destruction de celui-ci, dans le cas d'ablation complète, peut compenser le déficit de tonus provenant de l'enlèvement des lobes latéraux. D'autre part, la destruction du cervelet n'influence-t-elle pas la qualité plastique du tonus ? Les expériences de De Kleyn et de Magnus, étant des expériences d'ablations bilatérales, faites d'ailleurs dans un autre but, ne renseignent pas sur ces points. Seules, des ablations unilatérales pouvaient en décider, à condition de respecter, autant que possible le vermis.

Les expériences de Thuele (14) et de Weed (18) ont donné des résultats contradictoires quant au rôle des noyaux rouges dans le maintien de la rigidité. K. WiLson (19), dans son intéressante 
revue de la question, admet leur importance en se basant sur le fait qu'en les excitant électriquement, on obtient des réactions essentiellement toniques (Graham Brown. 8).

\section{La fonction inhibitrice du cervelet}

Des observations, dont les premières sont déjà anciennes, tendent à prouver qu'il existe dans le cervelet une fonction inhibitrice du tonus des extenseurs. SHERRINGTON (15) le premier, et bientôt après, LOEWENTHAl et HoRsLey (20) ont vu que l'excitation électrique du lobe antérieur inhibe régulièrement la rigidité de décérébration et que les effets obtenus sont surtout homolatéraux. THIELE (14) rapprocha ce fait de ceux qu'il observa lui même et qui en sont la contre-partie : chez l'animal intact, la lésion du lobe antérieur et la section des pédoncules cérébelleux supérieurs produisent une rigidité d'extension analogue à la rigidité de décérébration, mais plus faible. WEED (18) remarqua que chez l'animal décérébré l'ablation du lobe antérieur est régulièrement suivie de l'exagération extrême de la contracture. S. CoвB, A. BAiley et P. Holtz (21), à la suite d'expériences d'excitation, assignèrent aux influx inhibiteurs la voie olivo rubrale, mais ils mirent fortement en doute l'intervention du cortex cérébelleux dans le phénomène, laquelle avait été admise plus ou moins implicitement par les auteurs précédents.

On sait d'ailleurs que HoRSLEY et ClaRKE (22), après une révision critique des expériences d'excitation électrique du cervelet, sont arrivés à la conclusion que son écorce est inexcitable (dans le sens de ce mot pour l'écorce cérébrale) et que les effets attribués jusqu'alors à son excitation étaient dûs à des erreurs d'interprétation (diffusion de courant). Leurs conclusions ont été généralement acceptées et l'inexcitabilité de l'écorce cérébelleuse est devenue une notion classique. Les critiques de Horsley et CLARKe dans les cas particuliers qu'ils ont examinés, sont certainement justifiées mais lęurs expériences ont porté sur l'animal non décérébré, soumis à la narcose, et ne présentant pas par conséquent la rigidité qui met les inhibitions en évidence. (Il est surprenant que Horsley qui fut l'un des premiers à décrire les phénomènes d'inhibition, obtenus par l'excitation du lobe antérieur sur l'animal décérébré, n'en ait plus fait aucune mention dans son travail avec CLARKE, dont il est vrai que la première partie seule a été publiée). 
Quoi qu'il en soit, cetíe fonction d'inhibition du cervelet n'a pas fait jusqu'à présent l'objet d'une investigation myographique systématique et sa signification n'est pas claire.

Les recherches que j'ai effectuées se subdivisent donc en deux parties; dans la première. je me suis proposé de déterminer si l'ablation d'un lobe latéral du cervelet, c'est-à-dire des parties paires du néoet du palaeo-cerebellum, se traduit du côté opéré par une diminution de l'intensité de la contracture de décérébration, ou par une altération de sa plasticité étudiée myographiquement. J'ai étudié ensuite le mécanisme inhibiteur du vermis en m'attachant à préciser les points suivants, encore indéterminés : la participation du cortex cérébelleux dans les inhibitions, en d'autres termes, son excitabilité électrique ; la délimitation de la zone excitable et la signification éventuelle de ces limites ; l'action des inhibitions sur les fléchisseurs et sur l'activité spinale en général ; les voies aftérentes et efférentes des influx inhibiteurs ; enfin.la signification biologique de ce mécanisme et ce que l'on peut en déduire au point de vue de la pathologie du cervelet.

De plus, au cours de mes expériences, j'ai souvent pratiqué des transsections du tronc cérébral en arrière des noyaux rouges, ce qui m'a permis d'observer l'effet de leur exclusion sur la rigidité.

\section{Méthodes}

Les expériences ont porté sur 44 chats et 1 singe (Macacus Rhesus). Les ablations cérébelleuses aseptiques ont été faites à la curette après délimitation préalable par une incision sagittale. Les chats ont été décérébrés au moyen de l'appareil de MiLLER et SHERRINGTON (23). Les transsections ont été faites systématiquement antérieures, de façon que leur plan' passât en avant des tubercules quadrijumeaux et immédiatement en arrière des tubercules mammillaires. Pour les expériences d'excitation, la tente est enlevée et la découmerte du cervelet se poursuil aisément en arrière.

Différents muscles extenseurs et paires d'antagonistes ont été préparés pour l'enregistrement graphique, après paralysie des autres muscles du membre. Dans le cas du vasto-crural et du triceps brachial, l'enregistrement s'est fait par l'intermédiaire de l'attache osseuse du muscle ; les autres muscles étaient détachés en enlevant une lamelle osseuse de façon à respecter autant que possible les or- 
ganes sensitifs du tendon (SherRington). L'animal était immobilisé dans une position dorsale ou ventrale, symétrique. Les excitations électriques ont été faites à l'aide d'une bobine ordinaire sans graduation spéciale ; l'intensité du courant était évaluée par la sensation produite par l'application des électrodes sur la langue et par la distance du secondaire. L'intensité du primaire ne dépassait pas 0.2 Ampère. Electrode monopolaire (électrode stigmatique de SHERRINGTON) ou bipolaire (électrodes de platine distantes de $2 \mathrm{~mm}$.)

\section{Nomenclature}

J'ai adopté la nomenclature anatomique de IngVar (13) qui d'ailleurs est sensiblement la même que celle de Bolk (11) bien que la façon dont ces deux auteurs interprètent la morphologie du cervelet diffère essentiellement. La conception d' INGVAR est basée sur la comparaison des cervelets de mammifères avec ceux des reptiles et des oiseaux. Il reconnait trois divisions principales dans le cervelet des mammifères : (fig. 1) : $1^{\circ}$ le lobe antérieur (identique à celui de`Bolk),

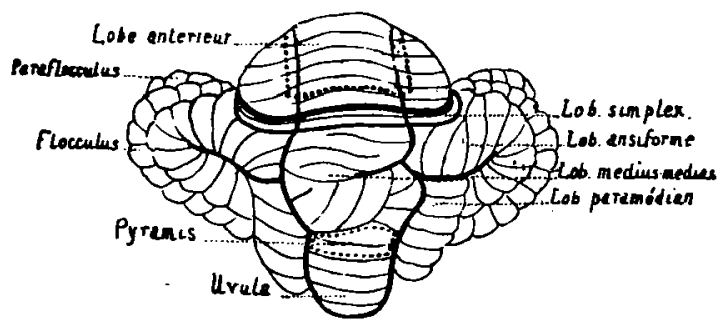

Fig. 1. - Le cervelet du chat ; face supérieure (d'après la nomenclature d'INGVAR). Le pointillé délimite les zones inhibitrices.

comprenant d'avant en arrière trois lobules : la lingula, le lobulus centralis et le culmen. Ce lobe est une formation médiane et impaire située en avant du sillon primaire $2^{\circ}$ le lobe moyen, qui contient les parties phylogénétiquement les plus récentes; il comprend deux structures impaires, le lobulus simplex et le lobulus médius medianus, et deux structures paires, les lobes a nsiformes et paramédians (qui ensemble forment le néo-cerebellum d'EDINGER); $3^{\circ}$ le lobe postérieur formé d'une partie médiane (pyramis, uvula, nodulus) et de parties latérales, le flocculus et le paraflocculus. Le lobe antérieur et le lobe 
postérieur constituent ensemble le palэeo-cerebellum qui comprend un étage vestibulaire (lingula, uvula, nodulus) et un étage spinal

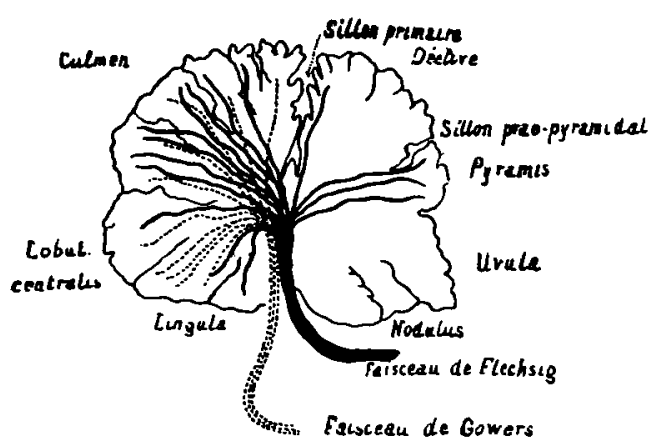

FIG. 2 A.

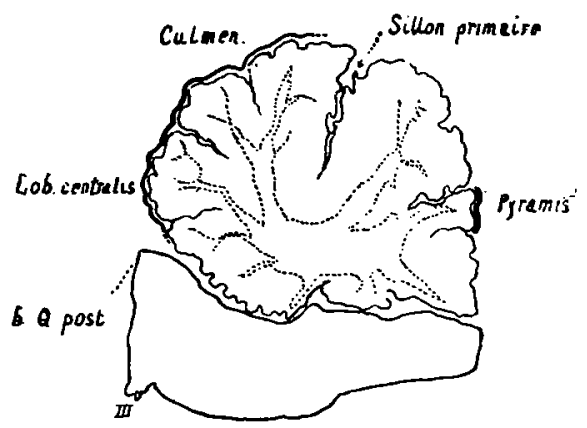

FIG. 2 B.

Coupe sagittale médiane du cervelet du chat Chat 41. Coupe sagittale à 2 millimètres à (schématique). Distribution des fibres spino- gauche de la ligne médiane. Les zones cérébelleuses (d'après INGVAR). excitables sont marquées par le trait plein qui double le contour.

(lobulus centralis, culmen, pyramis). L'existence de ces élages explique la division des fibres spino-cérébelleuses en deux groupes, antérieur et postérieur, telle qu'elle apparaît sur une coupe sagittale médiane (fig. 2, A.)

\section{I. - Le cervelet fournit-ll un appoint à la rigidité de décérébration?}

J'ai pensé qu'il était préférable de faire l'expérience en deux temps, car en faisant dans la même séance l'ablation cérébelleuse et la décérébration, on s'expose à de nombreuses causes d'erreur (choc, hémorragie, irritation).

Le lobe latéral gauche du cervelet (lobes ansiforme et paramédian, flocculus et paraflocculus ) a été enlevé aseptiquement à quatre chats. Tous les quatre ont présenté un hémi-syndrome cérébelleux typique avec, en plus, chez l'un d'eux, des symptômes vestibulaires transitoires. Sur l'un des animaux, très docile, il fut possible de prendre des tracés des réflexes rotuliens : ces tracés montrent, du côté cérébelleux, l'absence d'ondulation tonique, absence qui caractérise le réflexe dit pendulaire ( fig. 3 et 4). Ces quatre chats furent 
FIG. 3. - Réflexes rotuliens droit et gauche, pris sur l'animal non endormi, 3 jours après l'ablation du lobe gauche du cervelet. Les courbes se lisent de droite à gauche. Noter l'absence d'ondulation tonique sur les courbes du réflexe gauche. $1 / 100$ de sec. (réduit de $1 / 2$ ).
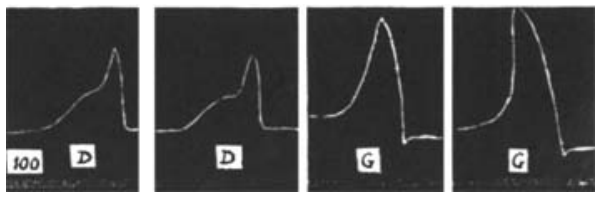

décérébrés respectivement les $3^{\mathrm{e}}, 8^{\mathrm{e}}$, $9 \mathrm{e}$ et $24^{\mathrm{e}}$ jours après l'opération. La rigidité des deux vasto-cruraux isolés a été comparée myographi-

Fig. 4. - Hémi-syndrome cérébelleux droit par blessure de guerre. Réflexe rotulien droit pendulaire. Réflexes droit et gauche iinscrits au moyen d'une ampoule de Marey, enregistrant le gonflement du muscle. La petite ondulation initiale $a b$ est due à la transmission de la percussion. Sa longueur correspond au temps de latence du réflexe. Remarquez la brièveté de la latence du réflexe droit (cérébelleux), l'amplitude beaucoup plus grande de la contraction, l'absence de l'ondulation tonique. 1/100 de sec. (réduit de moitié).
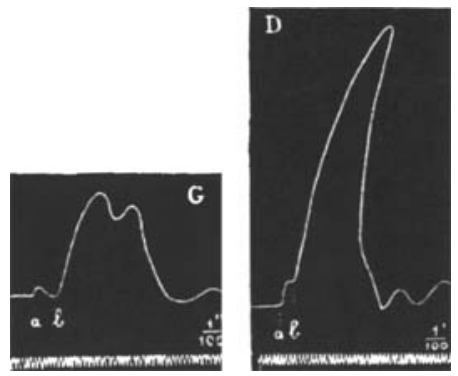

quement. Trois des animaux ont présenté une rigidité absolument égale quantitativement et qualitativement (plasticité) des deux côtés (fig. 5). Les courbes des réflexes rotuliens furent identiques.

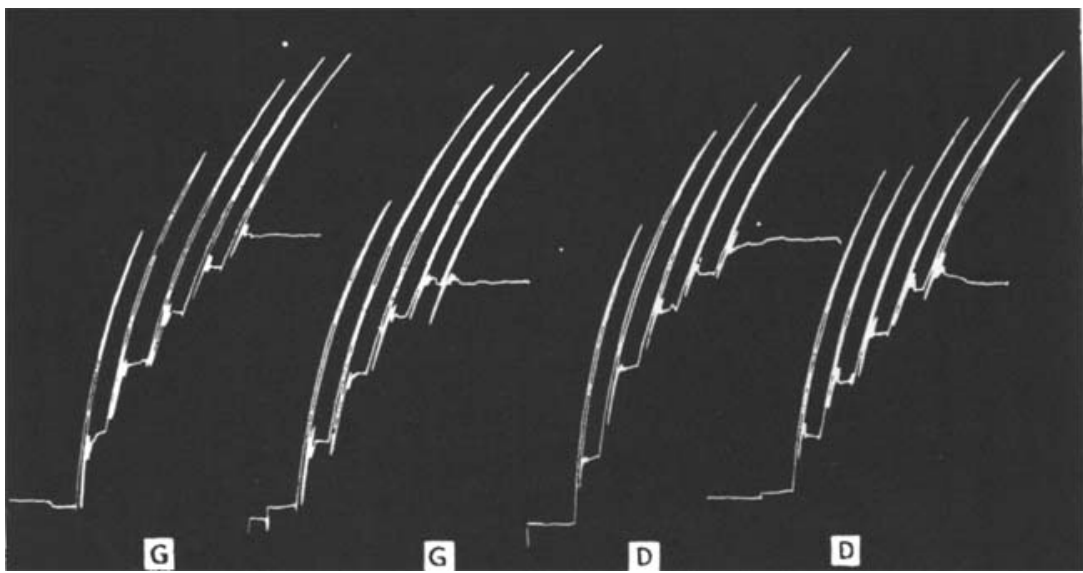

FIG. 5. - Lobe latéral gauche enlevé le 3-2-21, décérébration le 7-2-21, vastocruraux gauche $(G)$ et droit (D) isolés. Une série de 5 réflexes rotuliens élève le tonus de chaque muscle à la même hauteur, qui est maintenue : égalité de tonus et de plasticité. (réduit d'un tiers). 
Le quatrième eut une contracture légèrement plus marquée du côté cérébelleux. D'autre part, l'un des animaux présenta au bout de quelques heures une contracture de flexion qui, elle aussi, fut égale des deux côtés.

Autopsie. - Chat Il. Ablation complète du lobe latéral gauche. Intégrité du lobe antérieur et du vermis.

La transsection passe au milieu des tubercules quadrijumeaux antérieurs, à $1 \mathrm{~mm}$. en avant de l'émergence de III.

Chat III. Aḅlation incomplète du lobe latéral gauche. Les parties restantes du flocculus et du paraflocculus n'ont plus de connexion avec les noyaux centraux.

La transsection passe au milieu des T. Q. A., à $2 \mathrm{~mm}$. en avant de III.

CHAT V. Ablation complète du lobe latéral gauche et de la partie tout à fait latérale du lobe antérieur.

La transsection passe immédiatement en avant des T. Q. A. à l'émergence de III.

CHAT IV. Ablation complète du lobe latéral gauche et de la moitié gauche du lobe antérjeur et du vermis.

La transsection passe au milieu des T. Q. A., immédiatement en arrière des tubercules mammillaires.

Ces transsections ont donc respecté la voie olivo-rubrale et la plus grande partie, sinon la totalité des noyaux rouges.

Chez quatre autres chats, le cervelet fut enlevé aseptiquement aussi complètement que possible, et les animaux furent de même décérébrés quelques jours après. Tous les quatre présentèrent

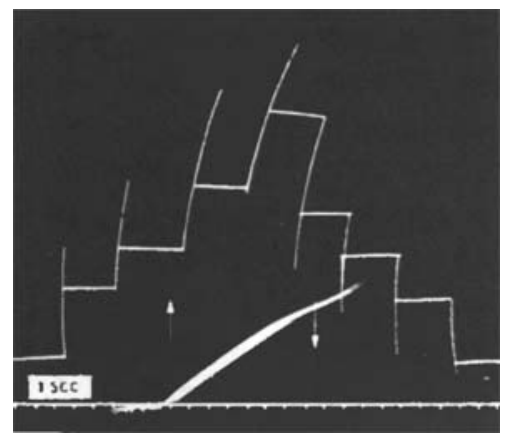

Fig. 6. - Chat 8. Ablation complète du cervelet et décérébration. Vasto-crural isolé. D'abord une série d'extensions, puis une série de flexions passives du genou, le tambour étant arrêté chaque fois. Montre la plasticité parfaite du tonus. Secondes. (Réduit de moitié).

une rigidité d'extension ; elle fut remarquablement plastique chez l'un d'eux (fig. 6). Or, l'ablation dans ce dernier cas était complète. 
Le cervelet ne fournit donc aucun appoint à la rigidité de décérébration.

Cependant, celle-ci résulte du fonctionnement d'un mécanisme postural statique libéré de ses inhibitions. Les muscles rigides sont ceux qui dans les conditions normales réagissent contre la pesanteur.

Il résulte de ce fait une espèce de paradoxe : un animal auquel un lobe latéral du cervelet a été enlevé est incapable de se tenir debout; si, en le tenant suspendu, on le rapproche du sol, les membres du côté opéré ne font pas la moindre tentative d'appui, bien qu'ils ne soient nullement paralysés : il semble que, de ce côté, soit détruit le mécanisme central par lequel se fait automatiquement la sustentation du corps. Le même animal, après décérébration, est pris d'un rigidité d'extension absolument égale des deux côtés ; posé sur le sol, il se tient debout (dans un équilibre évidemment précaire, puisqu'il n'est plus qu'une machine réflexe); et il n'est plus possible de déceler lequel des deux côtés est le cérébelleux.

Il faut donc en conclure que le cervelet ne contribue à la tonicité musculaire et ne participe au mécanisme de la station debout que par son association avec les centres supérieurs (thalamus, corps strié, écorce cérébrale).

On sait que, chez le lapin, le chat et le chien, le cortex cérébral et les corps striés ne sont pas essentiels au maintien de la station debout. L'intégrité du thalamus et du subthalamus suffit à assurer l'équilibre statique et chez l'animal thalamique le rapport des tonus des extenseurs et des fléchisseurs est normal [ Goltz (24), Dusser et Burenne (25), De Kleyn et Magnus (10) ].

Les recherches de DE KLEYn et MAGnus (10) ont déjà démontré que le cervelet n'est pas essentiel à l'exécution de certains des réflexes de redressemen، ("Stellreflexe ") qu'ils ont décrits el qui jouent vraisemblablement un rôle dans la statique normale. Mais il est certain que l'intégrité du cervelet est essentielle pour la synergie effective de ces différents réflexes. Supposer le contraire serait admettre qu'un animal cérébelleux, incapable de se tenir debout et de marcher, recouvrerait sa statique après enlèvement de ses hémisphères cérébraux. Chez l'homme, l'association du cervelet avec le cortex cétébral est certainement très importante et il n'est pas sans intérêt de noter que chez lui, la destruction de l'écorce motrice produit une hypotonie musculaire aussi marquée que l'hypotonie cérébelleuse.' 
En ce qui concerne le rôle des noyaux rouges, de nombreuses expériences me permettent de confirmer entièrement la conclusion négative de ThIELE (14) : les noyaux rouges ne fournissent aucun appoint à la rigidité ; au contraire, ainsi que cet auteur l'avait noté, leur exclusion l'augmente fortement ; la contracture, après une seconde transsection passant immédiatement en arrière des tubercules quadrijumeaux postérieurs et à la limite antérieure du pont, devient presque toujours extrême. La signification de ce fait deviendra claire dans la suite de l'exposé. Au cours de ces transsections successives du tronc cérébral, il peut survenir une hémorragie méningée qui comprime la protubérance et le bulbe, et abolit ainsi la rigidité. D'autre part, la section isolée des pédoncules cérébelleux supérieurs peut provoquer une abolition très transitoire de la rigidité, due sans doute à l'excitation par la section des fibres inhibitrices contenues dans ces pédoncules. Ces deux causes d'erreur expliquent sans doute les résultats discordants obtenus par certains auteurs.

\section{II. - Le Mécanisme inhibjteur du palaeocerebellum.}

Excitabilité du cortex du lobe antérieur. - Les excitations, faradiques de l'écorce du lobe antérieur produisent régulièrement l'inhibition de la rigidité de décérébration. L'écorce même est excitable; de nombreuses expériences de contrôle me l'ont démontré : une section parallèle faite à deux ou trois millimètres sous la surface ou le simple refroidissement, rendent la zone ainsi isolée ou paralysée inexcitable, même par des courants intenses, alors que l'excitabilité de l'écorce de la zone voisine intacte, n'a pas varié. L'intensité des courants requis est très faible, peut-être même plus faible que celle des courants nécessaires pour exciter l'écorce cérébrale (par exemple bobine à $20 \mathrm{~cm}$. pour une intensité du primaire de 0.2 Ampère). Ces courants sont généralement imperceptibles à la langue. Le seuil de l'excitation ne diffère pas suivant que l'on єmploie la méthode mono- ou bipolaire et j'ai pu m'assurer que par cette dernière méthode et dans les conditions de l'expérience, la pénétration effective du courant ne dépasse pas $2 \mathrm{~mm}$. de profondeur. Mais les effets ne sont obtenus que lorsque, malgré la décérébration et les manipulations qu'exige la préparation de l'animal, l'écorce est restée bien vascularisée et a conservé l'apparence luisante et 
rosée normale. Une écorce anémiée est toujours inexcitable. Dans ce cas, il est souvent possible d'obtenir des effets inhibiteurs en appliquant les électrodes dans le voisinage immédiat des pédoncules cérébelieux supérieurs ; mais les inhibitions ainsi obtenues diffèrent nettement de celles produites par l'excitation de l'écorce, en ce qu'elles sont accompagnées d'une contraction des fléchisseurs et notamment d'une flexion des griffes. L'application de compresses chaudes, presque brûlantes, sur l'écorce cérébelleuse, est très efficace pour maintenir ou rétablir son excitabilité, ainsi qu'il en est pour le cortex cérébral.

Limites de la zone excitable. - Pour la délimiter, j'ai fait usage des électrodes bipolaires qui évitent les erreurs dues à la diffusion. Quand la rigidité est faible, le "rebound "post-inhibiteur est le plus sûr témoin de l'inhibition.

La zone excitable a des lisnites précises (fig. 1 et 2 . B). Elle compprend le vermis antérieur, s'étend sur les côtés jusqu'à 1 millimètre environ à l'extérieur des veines qui le dessinent; en avant elle ne dépasse pas le milieu du lobulus centralis ; en arrière, elle s'arrête à 1 ou 2 millimètres du sillon primaire.

Il existe une seconde zone inhibitrice moins importante au niveau de la pyramis. Les inhibitions produites par son excitation sont moins marquées et nécessitent une intensité de courant un peu plus grandes que celle requise pour la zone du lobe antérieur. Pour cette raison et aussi parce qu'elle est plus difficilement accessible sur l'animal décérébré, mes expériences ont porté presque toutes sur le lobe antérieur. Le reste de la surface cérébelleuse est inexcitable dans les conditions de l'expérience.

Il faut signaler ici une coincidence remarquable : la topographie de ces deux zones excitables correspond exactement aux deux territoires de réception des fibres proprio-ceptives spino-cérébelleuses, ainsi qu'ils ont été établis par des travaux concordants de MAC Nalty et Horsley (27) et récemment IngVar (13), chez le chat.

Il résulte de leurs recherches que les fibres spino-cérébelleuses (faisceaux de Gowers et de Fleschig) se divisent à leur entrée dans le cervelet en deux contingents d'importance très inégale ; le premier, de beaucoup le plus nombreux, se distribue dans les parties médianes du culmen et de la moitié postérieure du lobulus centralis ; le second, 
qui ne comprend que quelques fibres, dans la pyramis (fig. 2, A). La coincidence entre les limites des zones inhibitrices et celles des zones spinales se vérifie jusque dans les détails : les deux lamelles postérieures du culmen ne recevraient pas ou presque pas de fibres; je les ai toujours trouvées inexcitables.

Ainsi l'inexcitabilité de l'écorce cérébelleuse ne peut plus être considérée comme un fait absolu. J'ai déjà fait remarquer qu'il 'était prudent de ne pas généraliser les conclusions de CLARKE et de HorSLEY (22), quelque motivées qu'elles soient, quand aux phénomènes particuliers que ces auteurs ont envisagés. En ce qui concerne les parties médianes du palaeo-cerebellum et en prenant comme test l'inhibition de la rigidité de décérébration, l'écorce cérébelleuse est excitable. Les cellules de Purkinje y son ${ }^{4}$ les seules neurenes à axone efférent. C'est donc elles qui, selon toute vraisemblance, transmettent les influx inhibiteurs. L'expérimentation met ici en évidence la fonction motrice qui résulte de la considération de leur structure. Bien entendu nous restons dans l'ignorance complète de la nature des influx efférents qu'émettent les cellules de Purkinje du reste du palaeo-cerebellum et de celles du néo-cerebellum tout entier. De même que nous ignorons aussi la nature des influx efférents que transmettent les cellules pyramidales des zones d'association du cortex cérébral. Malgré cette limitation, le fait me parait important en ce qu'il diminue le mystère de la structure et des fonctions de l'écorce cérébelleuse.

Il peut paraître étrange qu'à une délimitation anatomique et fonctionnelle aussi nette des zones inhibitrices, ne corresponde aucune différenciation histologique connue du cortex cérébelleux. Mais en somme, à part pour la frontale ascendante, les différences histologiques des circonvolutions cérébrales sont subtiles, et leur uniformité structurale fait le même contraste avec la spécialisation tranchée que révèle l'étude de leurs connections et de leurs fonctions : il faut être un spécialiste de la cyto-architectonique cérébrale pour reconnaître une préparation de l'écorce pariétale d'une préparation de l'écorce frontale.

La spécialisation fonctionnelle, dans le système nerveux, ne dépend que des connexions terminales des neurones. Elle peut exister, à un degré marqué sans se traduire par des différences structurales appréciables de ceux-ci. Les cellules pyramidales de la frontale ascendante sont en connexion avec les cellules de la corne antérieure : 
cette connexion détermine toutes leurs fonctions. Les grandes dimensions de ces neurones ne sont probablement que le résultat de leur fonctionnement moteur. On peut donc s'attendre à ce que les axones des cellules de Purkinje de la zone inhibitrice, aient des terminaisons spéciales. Toutefois nous l'ignorons encore. Mais nous savons déjà que les fibres de projection du palaeo-cerebellum ne vont pas aux mêmes noyaux que celles du néo-cerebellum : elles se projettent sur les noyaux du toit tandis que les fibres du néo-cerebellum se projettent sur les noyaux dentelés. D'autre part, le palaeo-cerebellum reçoit les fibres du bulbe et de la moelle. II fait donc partie d'un système spino-médullo-cérébelleux, distinct du système cérébrocérébelleux du néo-cerebellum [CLARKE et HoRSLEY (28)]. Les données de l'anatomie structurale et celles de l'anatomie comparée se superposent.

Description des effets. - Les zones excitables se divisent en deux hémi-zones, droite et gauche dont l'excitation inhibe la rigidité des membres homo-latéraux. L'effet est bilatéral pour le cou, le tronc et la queue : il n'y a donc aucune inclinaison ni torsion du cou et du tronc. L'inhibition des membres antérieur et postérieur est à peu près simultanée. Les effets sur les membres contralatéraux sont variables suivaní les animaux : le plus souvent, inhibition beaucoup moins marquée, parfois, au contraire, augmentation de la rigidité (fig. 7 et 8).

FIG. 7. - Chat 19. Sus-épineux droit et gauche isolés. Monopolaire sur l'hémizone gauche. Bob. $11 \mathrm{~cm}$. Ici, comme dans les tracés suivants, l'élévation de la ligne du signal signifie excitation. Secondes. (Réduit de moitié).

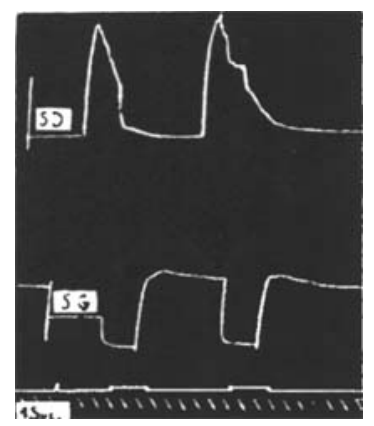

Les réponses contralatérales ne sont plus obtenues après section 
sagittale médiane du lobe antérieur (fig. 8). Cette section, par le

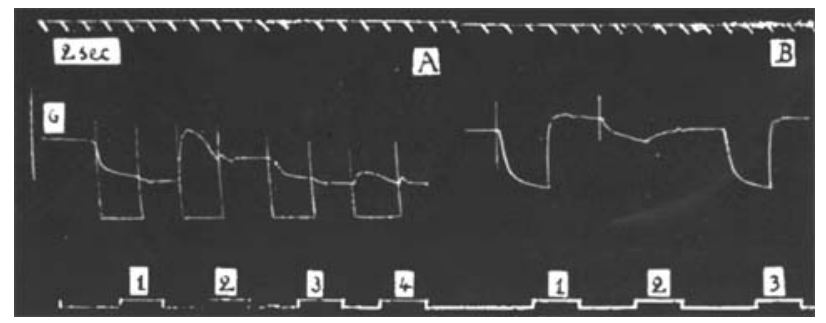

Fir. 8. - Chat 28. Gastro-cnémien gauche isolé.

A. 4 excit. bipolaires alternativement d'un point situé sur la veine gauche (1 et 3 ) et du point symétrique droit (2 et 4) Bob. $10.7 \mathrm{~cm}$.

B. Les 3 excitations suivantes sont faites 10 minutes plus tard, immédiatement après la section sagittale médiane du cervelet. Bob. $9 \mathrm{~cm}$.

1 et 3 même point sur la ve ine gauche; 2 , veine droite. 2 secondes. (Réduit de moitié).

traumatisne qu'elle comporte, diminue souvent l'excitabilité de la région, ce qui, en changeant les conditions de l'expérience, rend l'interprétation du résultat de ces sections sagittales délicate. L'existence de réponses contralatérales et leur moindre amplitude a peutêtre une relation avec le fait qu'un tiers environ de l'ensemble des fibres spino-cérébelleuses s'entrecroisent avant leur aboutissement au vermis antérieur d'après MAc NalTy et HorsLey (27).

Le temps de latence des réponses est très court.

Absence de localisation dans la zone excitable. - L'excitation d'un poinț quelconque de chaque hémi-zone inhibe également les membres antérieur et postérieur. De même, les fibres proprio-ceptives venant des membres antérieur et postérieur sont intimement mélangées dans chaque unité de surface du lobe antérieur [Mac Nalty et Hors. LEY (27)]. Ce qui est vrai pour les fibres afférentes doit sans doute l'être aussi pour les fibres efférentes. D'ailleurs MAC NALTY et Horsley, faisaient déjà remarquer que cette diffusion des influx afférents constitue un sérieux argument contre l'hypothèse de localisations cérébelleuses corticales.

J'ai pu vérifier sur un singe (Macacus Rhesus) que le phénomène y est essentiellement le même. C'est d'ailleurs sur le singe qu'il a été observé pour la première fois par SHERRINGTON (15). 
Action sur les fléchisseurs. - Les fléchisseurs ne sont influencés que d'une manière insignifiante par ces excitations de l'écorce cérébelleuse, soit dans le sens de l'innervation réciproque ordinaire sous forme de très faibles ondulations toniques (fig. 18), soil sous forme d'une inhibition simultanée à celle des extenseurs (fig. 9, 12, 13, 14, 19).

FIG. 9. - Chat 32. Triceps et Biceps gauches isolés. Inhibition d'un fléchisseur par l'excitation du cortex cérébelleux. $2 \mathrm{sec}$. (Réduit de moitié).

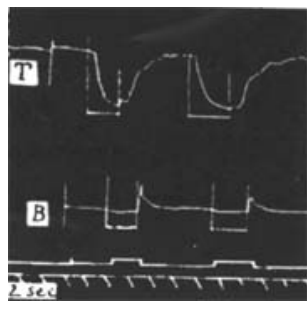

Les rebondissements qui suivent ces très faibles inhibitions, sont presque toujours très peu marqués. La courbe des fléchisseurs peut être tout à fait unie.

Cette absence d'action sur les fléchisseurs n'est pas due à leur manque de tonus, caractéristique chez l'animal décérébré. J'ai eu plusieurs fois l'occasion d'observer des animaux chez lesquels la contracture d'extension s'était transformée en contracture de flexion. Les excitations cérébelleuses, inhibitrices de la première étaient sans effet sur la seconde. Dans un cas spécial, le tonus était d'extension d'un côté, de flexion de l'autre; l'excitation cérébelleuse, qui inhibait régulièrement le tonus d'extension, n'influençait pas le tonus de flexion ; plus tard, au cours de l'expérience, ce dernier disparut et fut remplacé par un tonus d'extension identique à celui de l'autre côté. Les excitations cérébelleuses eurent alors sur lui leur effet inhibiteur ordinaire. L'autopsie dans ce cas montra que l'abolition transitoire du tonus d'extension était due à une hémorragie méningée unilatérale. Les réflexes auriculaires étaient abolis de ce côté.

Cette absence d'action sur les fléchisseurs distingue nettement ces réactions de celles obtenues par l'excitation des noyaux cérébelleux, des pédoncules cérébelleux supérieurs, des faisceaux longitudinaux postérieurs et des noyaux rouges.

Comparaison de l'inhibition cérébelleuse avec l'inhibition réflexe. L'ảbsence d'action sur les fléchisseurs différencie de même complè- 
tement les inhibitions cérébelleuses de celles obtenues par l'excitation d'un nerf périphérique. On sait que ces dernières ne sont qu'un des éléments d'une réaction complexe que l'on peut schématiser ainsi : du côté du nerf excité , contraction des fléchisseurs, inhibition des extenseurs ; de l'autre côté, inhibition des fléchisseurs, contraction des extenseurs.

J'ai voulu vérifier si cette différence n'était pas plus profonde encore et si l'inhibition cérébelleuse n'était pas uniquement une inhibition du tonus. Dans ce but,j'ai comparé l'action de ces deux modes d'inhibition sur l'amplitude et la forme d'un réflexe spinal, le réflexe contra-latéral d'extension. Je n'ai pu de cette façon trouver aucun critère permattant de les distinguer : sous l'influence d'une inhibition cérébelleuse, comme sous l'influence d'une inhibition réflexe, la réponse contra-latérale d'extension est diminuée à la fois dans son amplitude et dans son "after discharge "l'une étant en quelque sorte la mesure de sa composante phasique, l'autre de sa composante tonique. Il est vrai que ces deux composantes peuvent être influencẻes d'une façon très inégale, mais cela aussi bien par l'inhibition réflexe que par l'inhibition cérébelleuse (fig. 10 et 11) et il est difficile de
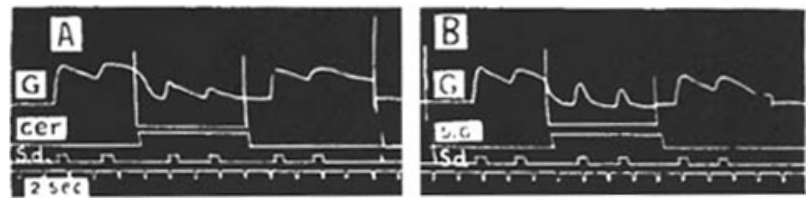

FIG. 10. - Chat 41. Gastro-cnémien gauche. Douze réflexes contra-latéraux d'extension.

A. Signal supérieur: excitation bipolaire de l'écorce du lobe ant. à 3 millimetres à gauche de la ligne médiane. Bob. $12 \mathrm{~cm}$. Signal inférieur : excit du sciatique popl. ext. droit. Bob. $20 \mathrm{~cm}$.

B. Signal supirieur : excit. du sciatique popl. ext. gauche, nerf inhibiteur. Bob. $22 \mathrm{~cm}$. Signal inférieur : excit. du sciatique popl. ext. droit, nerf excitateur. Bob. $20 \mathrm{~cm}$.

Noter en $A$ et $B$ la diminution de l'amplitude du réflexe contra-latéral d'extension. En A, l' " after-discharge * est partiellement conservée. 2 secondes. (Réduit de moitié).

déterminer la raison de cette électivité qui tantôt porte sur le tonus, tantôt sur le réflexe spinal. En général pourtant il semble bien que les inhibitions cérébelleuses dépriment spécialement le tonus, en respectant les activités spinales. Cela paraît résulter notamment de leur action sur les réflexes rythmiques de la progression. Mais le pait 


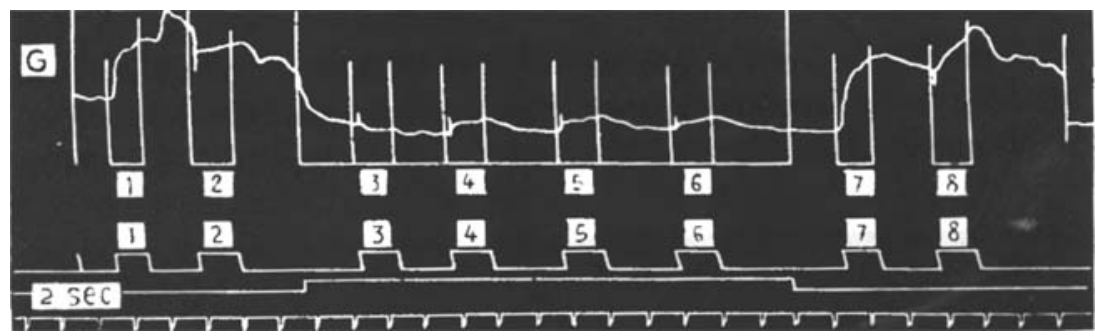

FIG. 11.-Chat 40. Gastro-cnémien isolé. Huit réflexes contra-latéraux d'extension

Signal supérieur : excit. du sciatique popl. ext. droit, nerf excitateur. Bob. $19 \mathrm{~cm}$. Signal inf. : excit. du sciatique popl. ext. gauche, nerf inhibiteur. Bob. $17 \mathrm{~cm}$. Montre la persistance de l' " after-discharge " du réflexe inhibé malgré la grande diminution de l'amplitude de celui-ci. (Réduit d'un tiers).

qu'elles peuvent déprimer aussi l'activité réflexe spinale donne lieu à une conséquence assez intéressante : les inhibitions cérébelleuses agissent aussi sur les neurones spinaux et non pas seulement sur les neurones toniques mésencéphaliques.

Action sur les réflexes rythmiques de progression. - Chez certains animaux décérébrés apparaissent des mouvements ayant le rythme et l'alternance de ceux de la progression. Cette tendance est due le plus souvent à un état d'asphyxie légère et prolongée. Il est intéressant d'observer l'action des excitations cérébelleuses sur ces mouvements. Cette action est, en apparence, très variable. Les excitations de l'écorce du cervelet peuvent provoquer ou inhiber ces mouvements ou modifier leur amplitude. Cette variabilité d'effet peut s'expliquer par l'existence d'un tonus optimum pour l'exécution des mouvements rytmiques de la progression, analogue au tonus oprimum du clonus. Par ex€mple dans la figure 12 , le niveau de tonus initial est bas. Une

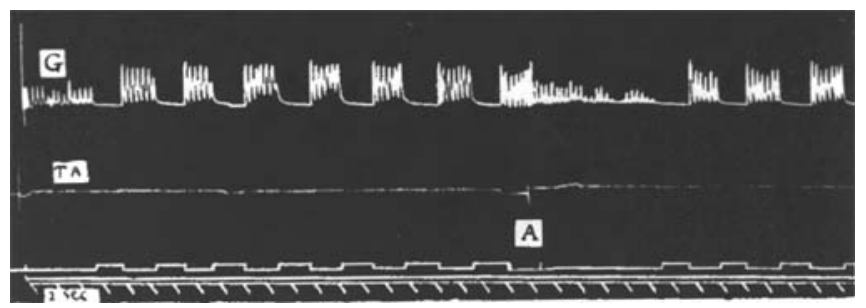

FiG. 12. - Chat 38. Gastro-cnémien et tibial antérieur gauches isolés. Electrode bipolaire sur l'hémi-zone gauche. Bob. $9 \mathrm{crn}$. Les mouvements de marche sont augmentés d'amplitude grâce à l'augmentation de tonus produite par le premier " rebound "; ils sont inhibés par les excitations cérébelleuses qui suivent. En A, tambour arrêté pendant 2 minutes. Les mouvements qui venaient de cesser sont reproduits à la faveur d'un nouveau "rebound ". (Réduit de moitié.) 
inhibition cérébelleuse l'élève par le rebondissement qui la suit. Les mouvements de marche qui sont ici des inhibitions rythmiques du tonus de l'extenseur augmentent d'amplitude et sont immédiatement abolis par des inhibitions cérébelleuses successives. La fig. 13 montre

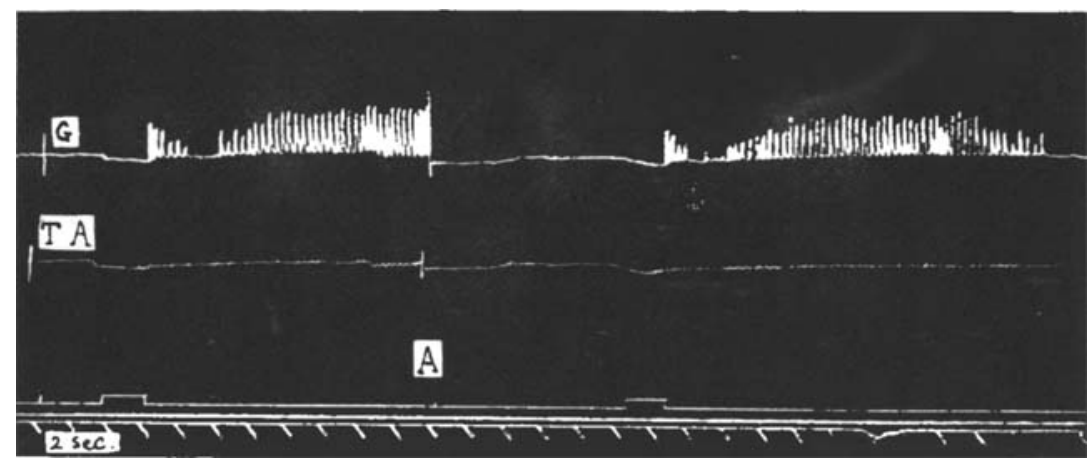

FIG. 13. - Chat 38. Mêmes dispositions que pour la figure 12. Les mouvements de marche apparaissent comme des inhibitions rythmiques du tonus, produites par le " rebound": la ligne fictive qui réunirait les sommets des battements rythmiques représente en réalité le niveau du tonus. En A, le tambour a été arrêté 2 secondes. (Réduit de moitié.)

deux séries de mouvements de marche, faisant suite chacune à une inhibition cérébelleuse. La fig. 14 montre bien l'existence d'un tonus

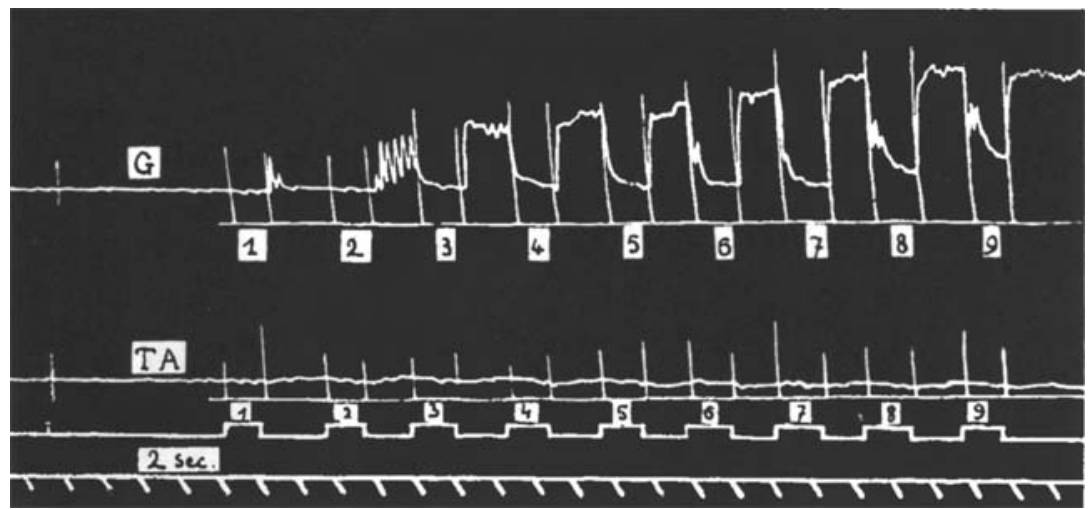

FıG. 14. - Chat 38. Mêmes dispositions que pour les fig. 12 et 13. Explications dans le texte. (Réduit d'un tiers.) 
optimum : au début, le tonus est nul, il n'y a pas de mouvements de marche. Ceux-ci apparaissent avec l'élévation du tonus, comme suite aux inhibitions 1 et 2 . Puis le tonus atteignant un niveau trop élevé, ils disparaissent, mais on les voit réapparaitre au cours même des inhibitions $6,7,8$ et 9 , lorsque le tonus, décroissant sous l'influence de ces inhibitions, atteint précisément le niveau optimum (comparer avec la fig. 15 qui montre la nécessité d'un tonus optimum pour

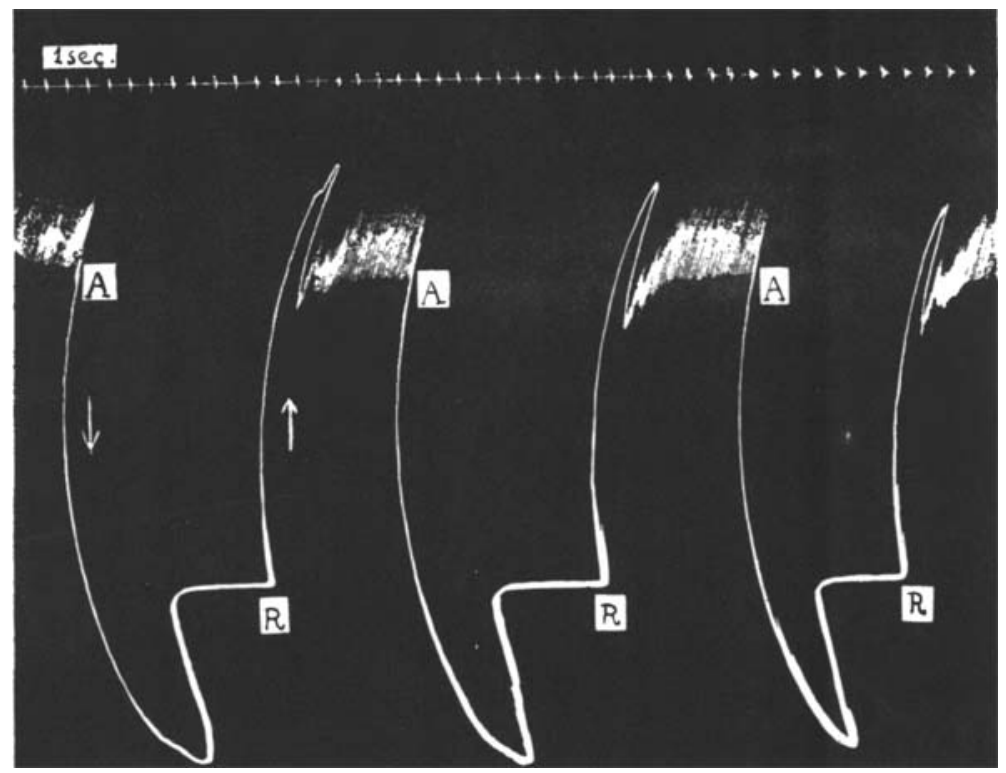

Fıg. 15. - Chat 13. Vasto-crural isolé. Clonus spontané. En A, production par flexion passive du genou d'une inhibition du tonus (réaction d'allongement). Le clonus cesse. En $R$, extension passive du genou . Rétablissement du tonus grâce à la plasticité : réapparition du clonus. (Réduit d'un tiers.)

l'entretien des mouvements d'un clonus). La preuve qu'il s'agissait bien, dans ces expériences, d'un tonus optimum, est donnée par le fait qu'il suffisait souvent de changer passivement le degré d'extension d'un membre intact pour provoquer ou abolir les mouvements de marche. MAGNus et DE KLEIN (7) avaient déjà̀ signalé que les mouvements de progression de l'animal décérébré sont influencés d'une façon très marquée par les variations de tonus des membres dépendant de la position des labyrinthes et du cou. D'ailleurs, 
l'importance des réflexes proprio-ceptifs comme adjuvants d'un facteur central, pour l'initiation et l'entretien des mouvements de progression, est bien établie depuis les travaux de SHERRINGTON. Il était intéressant de noter la facilité avec laquelle les excitations cérébelleuses semblent modifier ce facteur périphérique.

Le fait que les fléchisseurs ne participent généralement pas à ces mouvements rythmiques de progression, chez l'animal décérébré, est sans doute dî à leur absence de tonus.

Voies efférentes et afférentes. - La détermination des voies efférentes qui convoient les inhibitions, présente des difficultés spéciales à cause des particularités anatomiques de la région et surtout à cause de la constitution complexe des pédoncules cérébelleux supérieurs sur une partie de leur trajet. A priori, les voies efférentes des inhibitions semblaient devoir être des fibres cérébello-bulbaires : en effet, il résulte des recherches concordantes de A. Thomas (29) et de Clarke et Horsley (28) que le palaeo-cerebellum n'envoie aucune fibre aux noyaux dentelés, origine de la voie olivo-rubrale. Toutes ses fibres vont aux noyaux du toit. De ceux-ci partent des fibres, les unes directes, les autres croisées (fig. 20). Les fibres directes (fibres

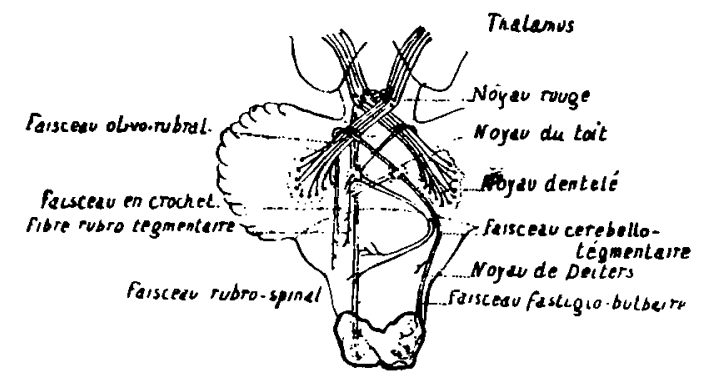

FIG. 20. - Schéma des voies efférentes du cervelet d'après EDINGE R (modifié). semi-circulaires, internes et externes), se terminent dans les noyaux vestibulaires. Los fibres croisées conitituent les faisceaux en crochet [VAN GEHUCHTEN (30)]. Ceux-ci, après s'être entrecroisés dans la substance blanche du cervelet, contournent les pédoncules cérébelleux supérieurs à leur émergence et descendent ensuite dans la formation réticulée du bulbe où ils s'épuisent. Il est probable mais non certain qu'une partie de leurs fibres se terminent dans les noyaux vestibulaires. Les faisceaux en crochet sont les seuls accessibles à l'expérimentation : la section médiane du cervelet les interrompt. Cette section ne supprime pas 
les effets inhibiteurs de l'excitation de l'écorce, bien que, à cause du traumatisme qu'elle comporte, elle puisse diminuer cette excitabilité. Celle-ci n'est pas modifiée quand la section s'est faite nettement sans hémorragie. D'ailleurs les faisceaux en crochet sont des voies croisées, tandis que les inhibitions sont surtout homolatérales.

Au contraire, la section du tronc cérébral, à partir d'un plan passant par le tiers postérieur des tubercules quadri-jumeaux postérieurs et le bord antérieur du pont, supprime immédiatement toute inhibition : ce plan exclut précisément les noyaux rouges. D'ailleurs CoBB, BAILEY et Holtz (21) ont contrôlé microscopiquement le niveau de leurs transsections et ont vu que les inhibitions n'étaient abolies que lorsque les noyaux rouges étaient complètement exclus. Malheureusement, ces auteurs excitaient directement les pédoncules cérébelleux supérieurs. Il m'est arrivé d'observer à la suite d'une transsection passant par un pareil plan, la conservation de l'inhibition des membres antérieurs, et l'abolition complète de celle des me mbres postérieurs dont la rigidité était devenue extrême. Des transsections aussi antérieures doivent avoir respecté les faisceaux en crochet. La section des pédoncules cérébelleux supérieurs abolit de même les inhibitions : la section d'un seul pédoncule (avant son entrecroisement) supprime les effets de l'excitation de l'hémi-zone homologue. L'excitation de l'autre hémi-zone continue à inhiber la rigidité de son côté ainsi que celle du tronc et de la queue.

Il faut donc admettre que les influx inhibiteurs empruntent une voie cérébello-rubrale. Ce ne peut être la voie olivo-rubrale, puisque le palaeo-cerebellum ne fournit pas de fibres aux noyaux dentelés. Ceci amène donc à envisager l'existence d'une voie fastigio-rubrale. L'existence de ces fibres, affirmée par certains, a été contestée par Van Gehuchten (30). Clarke et Horsley (28) ont vu, à la suite de la lésion d'un noyau du toit, la dégénération de certaines fibres du pédoncule cérébelleux supérieur homologue, mais il ne disent pas jusqu'où ils ont pu suivre ces fibres. Quoi qu'il en soit, l'existence d'un faisceau fastigio-rubral ne semble présenter à priori aucune impossibilité puisque la partie magno-cellulaire des noyaux rouges est phylogénétiquement très ancienne.

En raison du fait que les inhibitions dépriment l'activité spinale, il est vraisemblable que les influx empruntent ensuite une voie spinale (faisceau rubro-spinal) et non pas seulement des fibres tegmentaires. 
La concordance des zones inibitrices avec les territoires de réception des fibres spino-cérébelleuses permet de supposer que celles-ci sont les voies afférentes des réflexes inhibiteurs. La vérification expérimentale de cette hypothèse est très difficile. Il est vraisemblable que les réflexes inhibiteurs sont auto-régulateurs : or, on ne peut évidemment exciter sur la tranche de section proximale de la moelle épinière que les fibres spino-cérébelleuses provenant de muscles innervés par le segment distal de la moelle, L'excitation, sur la tranche de section proximale de la moelle dorsale et lombaire d'une zone étroite et superficielle correspondant au faisceau de Gowers, inhibe régulièrement la rigidité des membres antérieurs homo-latéraux. Il est probable que cette zone correspond à celle que FroenLich et SHERRINGTON (31) ont décrite, mais qu'ils ont située un peu moins superficiellement sur la tranche de section. Chez deux animaux, la transsection spinale fut faite au niveau de la onzième dorsale ; la zone inhibitrice correspondit exactement au faisceau de Gowers. Les effets inhibiteurs étaient d'ailleurs le plus facilement obtenus en plaçant l'éleetrode stigmatique sur la surface longitudinale de la moelle. Ils étaient régulièrement abolis par une égratignure superficielle en amont du point excité. Chez un troisième animal, la transsection passa au niveau de la deuxième lombaire. La zone inhibitrice siégea dans ce cas aux environs de la racine antérieure, comme dans les expériences de Froenlich et de SHERrington. L'existence de ces fibres inhibitrices provenant des membres postérieurs explique sans doute l'exagération extrême de la rigidité des membres antérieurs qui suit toute transsection spinale de la région dorsale ou lombaire chez l'animal décérébré. Mais ces inhibitions peuvent encore être produites après section des pédoncules cérébelleux supérieurs et des faisceaux en crochet. Il est donc probable qu'il existe dans le cordon de Gowers, comme Sherrington et Laslett (34) l'ont démontré pour le cordon de Flechsig, des fibres n'atteignant pas le cervelet $\left({ }^{(}\right)$. La présence dans le cordon de Gowers de ces fibres inhibitrices spino-spinales que l'on pourrait qualifier de directes, n'infirme pas l'hypothèse de la fonction inhibitrice d'une partie des fibres spino-cérébelleuses.

(1) Hосне et Thомаs (29) ont signalé la présence dans le cordon de Gowers de fibres longues d'association, mais MARBURG n'a pu se convaincre de leur existence (R. BING, 26, a, page 29). 
On ne peut faire état, pour ou contre cette hypothèse, des symptômes qui ont été observés à la suite d'une lésion expérimentale des faisceaux spino-cérébelleux sur l'animal intact. R. Bing (26) auquel on doit une étude très complète du.système spino-cérébelleux, a observé à la suite de la lésion haute du faisceau de Flechsig et d'une partie du faisceau de Gowers, un syndrome cérébelleux homo-latéral consistant en attitudes anormales, hypotonie, hypermétrie, sans oscillations du corps. Selon toute vraisemblance, les faisceaux spinocérébelleux sont de composition complexe et contiennent des fibres de fonctions différentes, les unes inhibitrices, les autres augmentatrices du tonus. D'autre part il faut tenir compte du fait que la spasticité qui, ainsi qu'on le verra plus loin, suit toujours les ablations du vermis, est un phénomène transitoire, ne durant que quelques jours. Cela est dû sans doute à des phénomènes de compensation.

Existence d'une inhibition tonique. - Le mécanisme inhibiteur cérébelleux a une fonction frénatrice permanente du tonus des extenseurs, fonction que l'on peut déceler aussi bien sur l'animal décérébré que sur l'animal intact.L'ablation, la lésion, le simple refroidissement du lobe antérieur, la section des pédoncules cérébelleux supérieurs, la transsection du tronc cérébral en arrière des noyaux rouges, sont régulièrement suivis d'une exagération, parfois extrême de la rigidité. Cette augmentation est unilatérale si la lésion ou la section l'est aussi. L'existence de cette fonction frénatrice explique sans doute aussi, comme l'avait déjà suggéré THIELE (14) la rigidité d'extension transitoire qui suit si fréquemment les ablations cérébelleuses sur l'animal intact. Cette rigidité est le plus marquée après les ablations du vermis. Le tableau que donne A. Thomas (33) des symptômes de la destruction totale du vermis, est celui d'une rigidité de décérébration : "... la tête est fortement inclinée en arrière, le tronc est incurvé dans le même sens, les membres extérieurs sont en extension forcée ; il se produit une chute à la renverse ". La rétropulsion si fréquemment notée est sans doute due à la même cause ; elle peut entraîner la progression rétrograde. $A$. Tномаs a produit les mêmes phénomènes par la destruction au moyen de l'anse galvanique du noyau du toit d'un côté : l'opisthotonos était marqué et le chat était entraîné en arrière et du côté de la lésion. Par contre, la section sagittale exactement médiane du cervelet n'est pas suivie de phénomènes spastiques et d'ailleurs produit très peu de symptômes (FERrier, Trendelen- 
BURG) (34). C'est là encore un argument contre l'hypothèse que les faisceaux en crochet seraient la voie suivie par les inhibitions $\mathbf{(}^{\mathbf{1}}$.

Je n'ai observé qu'un seul cas dans mes expériences d'ablation, où une rigidité transitoire n'ait pas paru et précisément dans ce cas le lobe antérieur avait été entièrement respecté.

Il est probable aussi que la rigidité, l'opisthotonos et les spasmes d'extension qui sont fréquemment observés chez les malades atteints d'une tumeur du cervelet, sont dus à la destruction ou à la paralysie transitoire de ce mécanisme inhibiteur cérébelleux. K. WILson (19) avait déjà donné cette interprétation à la rigidité d'un de ses malades.

La destruction du mécanicme inhibiteur cérébelleux ne produit chez le chat et le chien, qu'une rigidité d'extension beaucoup plus faible que la rigidité de décérébration. Chez ces animaux, l'activité inhibitrice permanente du cervelet est donc moins efficace que celle des noyaux subthalamiques et des corps striés. Mais il est possible que chez des mammifères plus primitifs, chez lesquels le palaeo-cere. bellum est relativement plus important, sa fonction inhibitrice soit plus importante aussi. Ainsi s'expliquerait le fait que chez le lapin, pour obtenir une rigidité de décérébration, il est nécessaire de faire la transsection du tronc cérébral à un niveau très postérieur situé en arrière des noyaux rouges (MAGNus) (36).

Réactions posturales cérébelleuses. - L'excitation des noyaux cérébelleux centraux et des pédoncules supérieurs produit des réactions, toniques posturales, qui mettent en action les fléchisseurs et les extenseurs. On ne peut réaliser correctement ces excitations qu'en employant, comme le firent HorsLey et ClaRke (22), des précautions spéciales pour éviter la diffusion et assurer une localisation exacte du courant excitant. La signification de ces réactions posturales cérébelleuses n'est pas claire. Elles ne sont pas sans analogie avec celles que produit, d'après Graham Brown (8), l'excitation des faisceaux longitudinaux postérieurs et des noyaux rouges. Dans mes expériences, $j$ 'ai noté que des mouvements lents de flexion (plus rarement d'extension) des griffes étaient l'indice d'une diffusion du courant aux pédoncules cérébelleux supérieurs.

(1) Cel? n'implique pas que les fibres cérébello-bulbaires ne puissent véhiculer (i'aitres inhibitions, et à ce sujet il est intéressant de noter avec MaGNus que les lésions du vermis sont régulièrement suivies d'une exagération des réflexes vestibulaires (BAUER et LEIDLER) (35)' 


\section{Conclusions}

En résumé, lorsque le cervelet est coupé de son association avec le thalamus et l'écorce cérébrale, il n'est pas possible de déceler son activité sous forme d'un appoint à la rigidité de décérébration ou d'une influence sur la qualité plastique de celle-ci. Le néo-cérebellum qui est la partie du cervelet associée à ces centres supérieurs, est en quelque sorte inerte et paralysé lorsqu'il est séparé des ses animateurs, par la décérébration. Par contre, le palaeo-cerebellum manifeste des activités propres. Son écorce a une fonction d'inhibition du tonus des extenseurs, prouvée à la fois par les résultats de son ablation et de son excitation. La concordance exacte des zones inhibitrices avec les zones spinales, proprio-ceptives, du cervelet, indique que le mécanisme est une auto-régulation. L'utilité d'une régulation $\mathrm{du}$ tonus par des centres de corrélation supérieurs aux centres spinaux semble clairement montrée par la nécessité d'un tonus optimum pour l'entretien des mouvements rythmiques de la progression.

Il est possible que l'hypermétrie des cérébelleux soit due à une destruction de ce mécanisme auto-régulateur. Les recherches de A. Troell et de C. Hosser (12) montrent que c'est à la suite des ablations du lobe antérieur chez le chat que l'hypermétrie et les mouvements impulsifs sont le plus marqués.

L'existence d'une fonction d'inhibition permanente du tonus des extenseurs et la multiplicité des centres qui paraissent assumer cette même fonction (pallidum, subthalamus, écorce du palaeo-cerebellum) s'expliquent sans doute par la nécessité d'une dépression constante de ce tonus, que la station debout et les tractions des extenseurs qu'elle comporte, tendraient sans cesse à exagérer ( ${ }^{\mathbf{1}}$ ).

(1) Si cette hypothèse est correcte, cette fonction inhibitrice n'est pas nécessaire chez les mammifères aquatiques et on peut s'attendre a ce que le lobe antérieur du cervelet présente de ce fait des variations d'importance. Or, le lobe antérieur des cétacés forme précisément une exception remarquable dans l'anatomie du cervelet des mammifères [BoLK (11)]. Chez tous les mammiferes terrestres, le lobe antérieur montre une grande homogénéité de structure et est limité en arrière par le sillon primaire, très profond. Chez les cétacés, on ne trouve pas ce sillon primaire et il est impossible de délimiter un lobe antérieur ; en outre, la partie antérieure du cervelet des cétacés est pourvue de parties hémisphériques absentes dans le lobe antérieur des au tres ordres de mammifères. Tous ces détails, décrits par BoLK, m'ont été aimablement confirmés par le professeur ARİENS KAPPERS. 
Cette fonction régulatrice du tonus, que manifeste le palaeo-cerebellum, pourrait donc être active indépendemment des associations que le néo-cerebellum a contractées avec le thalamus et l'écorce cérébrale. Chez les vertébrés primitifs, le palaeo-cerebellum constitue la totalité ou la plus grande partie du cervelet. Les systèmes moteurs que forment les associations du néo-cerebellum avec le thalamus et l'écorce cérébrale utilisent sans doute le mécanisme primordial du palaeo-cerebellum, en conformité avec le plan général du sytème nerveux. Il semble même difficile qu'il en puisse être autrement, car non seulement l'écorce du palaeo-cerebellum envoie des influx efférents, mais encore c'est elle qui reçoit tous les influx afférents, d'origine musculaire et vestibulaire. Mais dans l'ignorance complète où nous sommes encore de la nature des fonctions du néo-cerebellum et de ses connexions avec le palaeo-cerebellum, il est impossible de se faire une idée de ce qu'est leur synergie. L'absence de grandes voies d'association connues dans le cervelet ne facilite pas la solution du problème.

Les connexions qui sont la base de cette synergie se font peut-être dans les noyaux du toit. Ceux-ci sont un relai sur les voies efférentes du palaeo-cerebellum, mais ils reçoivent aussi, d'après CLARKE et HoRsLeY (28) des fibres de toutes les autres régions de l'écorce.

C'est l'analyse de cette synergie et de la synergie du cervelet avec les systématisations supérieures qui seule pourrá faire pénétrer plus avant dans la connaissance de sa physiologie.

D'autre part, l'existence dans le cervelet d'un mécanisme inhibiteur montre bien le danger en matière de physiologie cérébelleuse,des conceptions trop simplistes, quelque séduisante que puisse être cette simplicité. Certes l'hypotonie rend compte de bien des symptômes cérébelleux, comme l'a justement fait remarquer WALSH (37) dans un essai récent . La courbe d'un réflexe rotulien cérébelleux, comparée avec celle du réflexe normal (fig. 3 et 4), montre bien quelques-unes des conséquences de l'hypotonie sur la motricité : brièveté du temps de latence, amplitude plus grande de la contraction, absence d'ondulations toniques. Mais la simplicité de l'hypothèse me parait être fallacieuse quand elle veut ne faire que des corollaires de l'hypotonie de tous les autres signes du déficit cérébelleux dont nous devons l'individualisation à Lucianı, BABINSKI et A. ThомAs, notamment l'astasie, l'hypermétrie, la passivité. Pour ne parler que de cette dernière, $A$. Thomas et $G$. Holmes ont bien montré qu'elle dépend d'un manque de vigilance des antagonistes et non pas seulement de 
l'hypotonie. Aussi, en attendant une terminologie plus physiologique, devrons-nous nous contenter encore de cette terminologie qui n'est que descriptive, mais qui décrit bien.

\section{Annexe}

Il me reste à décrire quelques observations qui, bien que faites au cours de ces expériences, n'ont pas de relations directes avec la physiologie du cervelet. Elles touchent à des problèmes plus généraux : les unes concernent la complexité des réflexes spinaux; les autres, la nature encore discutée des phénomènes de rebondissement (urebound").

Complexité des réponses cérébelleuses obtenues par l'excitation simultanée des fibres droites et gauches. - Cette expérience n'a d'intérêt que dans les cas où les réponses homo- et contra-latérales sont de signe différent, c'est-à-dire inhibition latérale et augmentation contra-latérale.

Cette excitation simultanée est réalisée le plus aisément grâce à l'exiguité de la région, en employant une électrode monopolaire appliquée sur la surface du cervelet, avec des courants supra-liminaux; de cette manière, les fibres droites et gauches sont excitées simultanément dans la profondeur. Dans ce cas, on obtient, si l'on enregistre le tonus d'un muscle extenseur, des réponses complexes consistant au cours de l'excitation en une alternance d'effets inhibiteurs et augmentateurs. On réalise ainsi une véritable incoor-

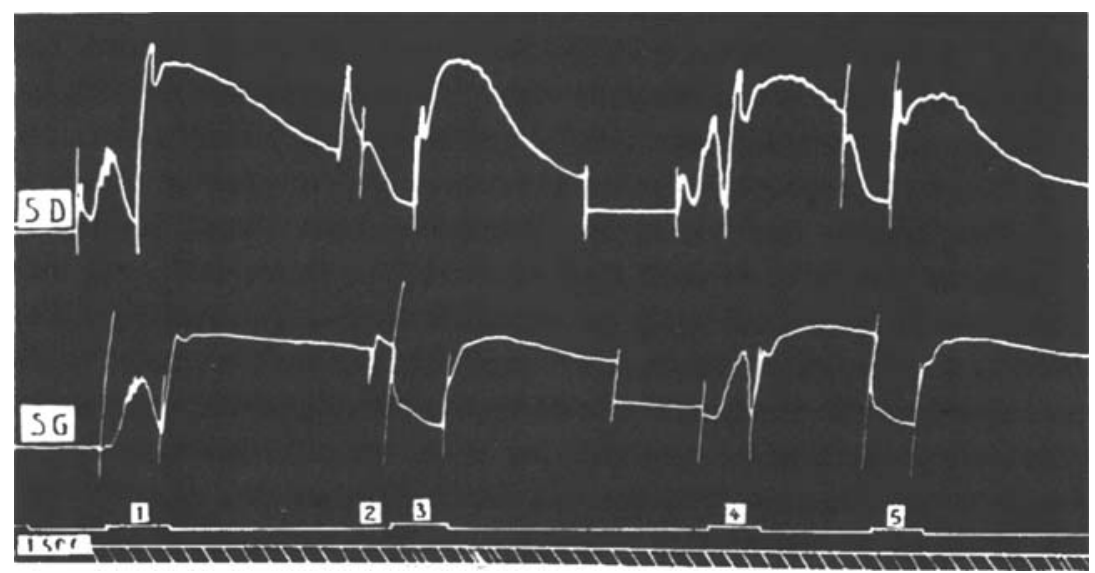

FIG. 16. - Chat 19. Suscapulaire isolé. Monopolaire $7 \mathrm{~cm}$. sur ligne médiane. Secondes. Explication dans le texte. (Réduit d'un tiers.) 
dination (fig. 16). Il est possible de varier à volonté la forme de la réponse en déplaçant simplement l'électrode de la ligne médiane vers les côtés. Une réponse qui paraît purement augmentatrice peut traduire sa composante inhibitrice par un rebondissement d'extension (rebound) à l'interruption du courant (fig. 17 B). Il peut
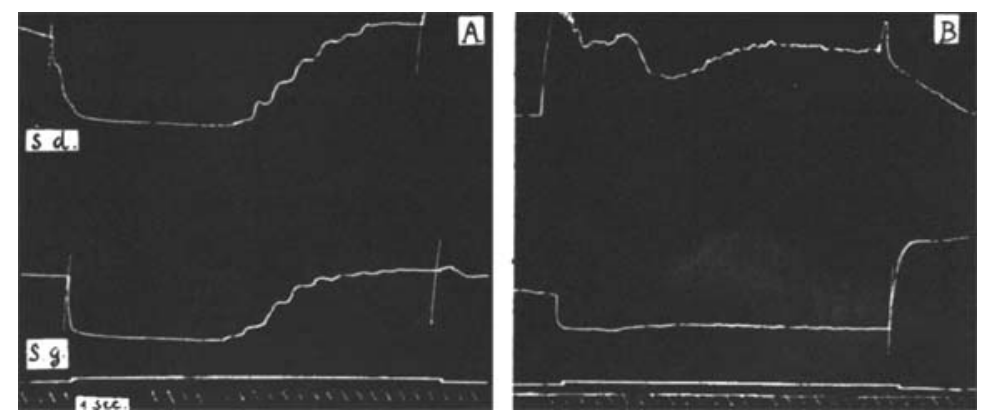

FIG. 17. - Chat 19. Monopolaire $7 \mathrm{~cm}$. $A$, sur ligne médiane; $B$, à 3 millimetres à gauche de la ligne médiane. Secondes. (Réduit de moitié.)

se faire, ainsi que le montre la figure 16 , que la forme de la réponse dépende de l'état de tonus initial du muscle. Une excitation qui produit sur le muscle atonique un mélange d'augmentation et d'inhibition donne, aussitôt que ce muscle a repris de la rigidité, une inhibition pure, pour les mêmes conditions d'expérience. On sait que le même phénomène d'inversion ("Umkehr "), se produit dans l'excitation d'un nerf périphérique. L'un des plus intéressants parmi ces effets complexes est le suivant (fig. 17) : en plaçant l'électrode stigmatique exactement sur la ligne médiane, il est possible d'exciter à la fois et en proportions égales les fibres droites et gauches. Dans ce cas, tout comme dans le cas de l'excitation exactement équilibrée [SHERRington (38)] de deux nerfs symétriques, l'effet initial est une inhibition bilatérale des muscles extenseurs droits et gauches (fig. 17A) Mais si le passage du courant est prolongé pendant un nombre de secondes variant de vingt à quarante, on voit réapparaitre des deux côtés une extension progressive. Au cours de cette réascension du tonus se produisent parfois des ondulations rythmiques (fig. $17 \mathrm{~A}$ ). Au moment où l'excitation cesse, un " rebondissement d'extension" peut révéler la persistance de l'action inhibitrice. Il est probable que 
cette forme particulière d'alternance est due à une fatigue plus rapide de l'inhibition, permettant à l'effet augmentateur de dominer [Forbes (39) a montré qu'il existe une fatigue de l'inhibition]. La courbe $B$ prise quelques minutes après la courbe $A$ (fig. 17) montre que le phéncmène est bien dû à l'excitation de fibres de fonctions différentes. Ici l'électrode est placée à 3 millimètres à gauche de la ligne médiane : le sus-épineux gauche subit une inhibition maintenue (excitation des fibres inhibitrices gauches) tandis que le droit montre une augmentation de tonus mêlée d'une inhibition qui se révèle par les ondulations de la courbe et le "rebound " consécutif (excitation des fibres augmentatrices provenant de l'hémi-zone gauche, plus excitation par diffusion de fibres inhibitrices droites).

La complexité de ces réactions ressemble fortement à la complexité des réponses réflexes obtenues par l'excitation de nerfs périphériques qui ont été décrites si minutieusement par SHERrington et Graham Brown.

La fig. 11 montre des exemples de cette complexité. Une série de réflexes contra-latéraux d'extension sont provoqués par des courants d'intensité égale; les réponses 1 et 6 données par le muscle atonique consistent en une extension pure ; les réponses 2 et 8 données par le muscle en état de tonus débutent par une inhibition: c'est le phéno. mène d'inversion décrit plus haut. Noter également la complexité des reponses $3,4,5$ et 6 .

On n'est pas encore d'accord sur l'interprétation qu'il convient de donner à la complexité des réflexes périphériques.

Les uns, comme Sherrington (40), l'attribuent à l'excitation dans le nerf, de fibres de fonctions différentes; d'autres, comme A. Forbes (40) tentent de l'expliquer, sur la base de l'inhibition de WedenskI, par des variations de fréquence des trains d'ondes nerveuses, fréquence alternativement inhibitrice et excitatrice. Le fait que, dans le cas des excitations cérébelleuses, des réponses complexes ne sont obtenues que lorsque des fibres de fonctions différentes sont excitées simultanément, me paraît être un argument en faveur de l'hypothèse de Sherrington. En effet, les réponses obtenues soit avec l'électrode monopolaire en employant des intensités liminales, soit avec les électrodes bipolaires dont l'action est toute superficielle, sont toujours simples, purement inhibitrices ou augmentatrices (fig. 7, 8, 9, 10, $12,13,14,18,19$.) 
Phénomènes de rebondissement ("rebound"). - Les inhibitions cérébelleuses sont généralement suivies d'un rétablissement brusque du tonus d'extension ("rebound »). Celui-ci montre souvent un phẻnoméne de facilitation à la suite d'excitations brèves successives (fig. 14 et 18). Cette facilitation porte à la fois sur sa latence et sur son ampli-

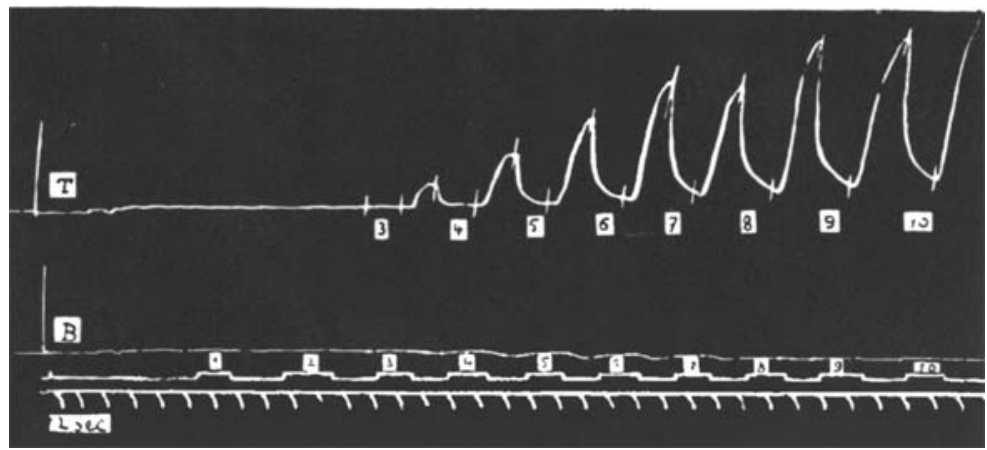

FIG. 18. - Chat 34. Triceps et Biceps gauches isolés. Les électrodes bipolaires sont maintenues appliquées à 3 millimetres à gauche de la ligne médiane. Bob. à $8 \mathrm{~cm}$. pour toutes les excitations. Facilitations du rebound : les excit. 1 et 2 sont sans effet apparent (tonus nul), l'excit. 3 est suivie d'un " rebound " faible après un long retard, le tonus produit par le rebound est inhibé à son tour par l'excit. 4 qui est suivie d'un rebound plus marqué après une latence moindre. Les rebounds successifs sont de plus en plus amples, leur latence de moins en moins longue. (Réduit de moitié environ.)

tude. Elle dépend d'un phénomène central et non pas d'une facilitation du processus d'excitation du cortex cérébelleux : il est possible d'abolir par une inhibition prolongée, un " rebound " qui vient d'être facilité et si l'on reproduit alors de nouvelles inhibitions brèves, les rebondissements qui les suivent montrent à nouveau le phénomène de facilitation. L'existence de cette facilitation n'est pas sans intérêt à cause de l'incertitude qui règne encore sur l'interprétation qu'il convient de donner aux phénomènes de " rebound ". BERITOFF (42). a proposé l'hypothèse de leur nature réflexe. Il se base sur le fait que, chez l'animal décérébré, l'excitation d'un nerf périphérique quelconque est capable de déclancher une réaction tonique latente, préparée et déterminée par la position des labyrinthes et du cou. Le rebound serait la manifestation d'un pareil réflexe tonique vestibulaire, à la fois provoqué et inhibé par l'excitation du nerf et ne pouvant, par conséquent, se manifester qu'à la cessation de cette excitation. 
L'existence d'une facilitation centrale du rebound plaide évidemment en faveur de l'hypothèse de sa nature réflexe. La fréquence de cette facilitation par les excitations de l'écorce du lobe antérieur s'expliquerait par les connexions qui unissent cette écorce aux noyaux vestibulaires par l'intermédiaire des noyaux du toit. D'autre part, j'ai observé sur certains de mes tracés, des courbes qui montrent la complexité des phénomènes de rebondissement. Un rebondissement d'extension, en apparence simple, peut être en réalité formé de deux éléments qu'il est possible de décomposer. Le premier est un rétablissement du tonus, brusque ; c'est probablement le réflexe tonique vestibulaire de BERITOFF. Le second est une ascension plus lente $\mathrm{du}$ tonus (fig. 9, 16 et 19). Une inhibition prolongée peut ne pas diminuer
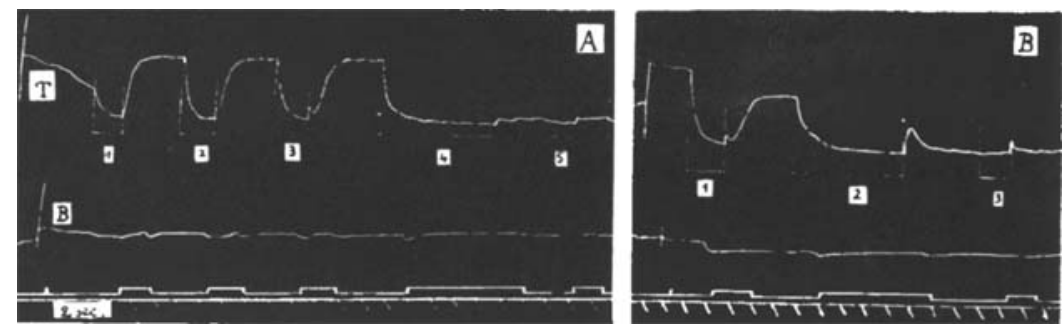

FIG. 19. - Chat 32. Triceps et Biceps gauches isolés. Electrode bipolaire à gauche de la ligne médiane, maintenue appliquée au même point. Bob. $9.8 \mathrm{~cm}$. Dédoublement du rebound. 2 secondes. Explication dans le texte. (Réduit de moit ié.)

ou même augmenter l'amplitude du premier élément et abolir le second (fig. 19, A. 4, et fig. 19, B. 2). Cette ascension plus lente du tonus a les allures d'une réaction de raccourcissement [巛 shortening réaction" de SHERRington (43)], c'est-à-dire donc d'un réflexe proprio-ceptif tonique. Elle se greffe sur la contraction de rebound. Il est intéressant de noter que cette fusion de deux contractions toniques dont l'une est engendrée par l'autre, peut être si intime qu'elle ne se traduit même pas par une ondulation de la courbe myographique (fig. 19, A. 1).

Je suis heureux de pouvoir exprimer ici toute ma reconnaissance au professeur Sir Charles Sherrington, pour ses critiques et ses conseils qui m'ont été si précieux au cours de mon travail, ainsi que pour la bienveillante hospitalité que j'ai reçue dans son laboratoire.

J'adresse aussi tous mes remerciements à mon ami le docteur PERcival Bailey qui a bien voulu me prêter le concours de son expérience chirurgicale pour les opérations d'ablation. 


\section{RESUME}

1. Le cervelet (écorce et noyaux centraux) et les noyaux rouges ne fournissent aucun appoint à la rigidité de décérébration et celle-ci n'est pas moins plastique après leur ablation.

2. L'écorce du palaeo-cerebellum est excitable chez l'animal décérébré : l'excitation du lobe antérieur (BoLk) par des courants très faibles inhibe régulièrement la rigidité de décérébration et les effets inhibiteurs sont immédiatement abolis par une section faite sous la surface active, ou par le simple refroidissement de celle-ci. Il existe une seconde zone inhibitrice au niveau de la pyramis.

3. Les zones excitables correspondent exactement aux deux territoires de réception des fibres spino-cérébelleuses.

4. Elles se divisent en deux hémi-zones, droite et gauche dont l'excitation inhibe la contracture d'extension des membres homolatéraux, et produit des effets contra-latéraux variables: inhibition moins marquée ou augmentation du tonus.

5. Il n'y a pas de localisation à l'intérieur de chaque hémi-zone.

6. Les fléchisseurs ne sont influencés que d'une façon insignifiante par ces excitations de l'écorce cérébelleuse, ce qui distingue nettement ces réactions de celles obtenues par l'excitation des noyaux dentelés et des pédoncules cérébelleux supérieurs.

7. L'absence d'action sur les fléchisseurs différencie aussi les inhibitions cérébelleuses des inhibitions réflexes, mais il n'existe pas d'autre critère permettant de distinguer ces deux modalités d'inhibition. Toutes deux diminuent à la fois l'amplitude et l' cafterdischarge " d'un réflexe contra-latéral d'extension. L'inhibition cérébelleuse porte donc aussi sur les neurones spinaux, et non pas seulement sur des neurones toniques du métencéphale.

8. Les excitations de l'écorce cérébelleuse ont des effets en apparence divers sur les mouvements rythmiques de progression que peuvent présenter les animaux décérébrés. Cette diversité peut s'expliquer par l'existence d'un tonus optimum pour l'initiation et l'entretien des mouvements rythmiques de la progression.

9. La voie efférente de l'inhibition paraît être une voie cérébellorubrale. Ce ne peut être qu'une voie fastigio-rubrale, dont l'existence pourtant n'est pas prouvée anatomiquement. 
La coĩncidence des zones inhibitrices avec les territoires de réception des fibres spino-cérébelleuses indique que celles-ci sont les voies afférentes des réflexes modificateurs du tonus. La vérification de cette hypothèse est très difficile. Il existe des fibres inhibitrices dans le cordon de Gowers, mais elles sont spino-spinales.

10. Le mécanisme inhibiteur du palaeo-cerebellum a une fonction frénatrice permanente du tonus des extenseurs : sa destruction est régulièrement suivie, chez l'animal normal, de la production d'une rigidité d'extension, et chez l'animal décérébré d'une exagération de la rigidité préexistante.

L'existence de cette fonction frénatrice explique les spasmes d'extension et la rétropulsion des ablations du vermis ainsi que les phénomènes analogues'dans les cas de tumeur du cervelet chez l'homme.

11. Le cervelet ne fournit donc son appoint à la tonicité musculaire que par son association avec le thalamus et l'écorce cérébrale (système cérébro-cérébelleuX de CLARKE et HoRSLEY).

Il existe dans le palaeo-cerebellum un mécanisme inhibiteur du tonus des extenseurs. Ce mécanisme correspond anatomiquement au système spino-cérébelleux. Il est auto-régulateur : la concordance des zones inhibitrices avec les zones proprio-ceptives du cervelet l'indique. L'utilité de cette auto-régulation est montrée par 'la nécessité d'un tonus optimum pour l'entretien des mouvements rythmiques de progression.

12. Dans le cas où les excitations cérébelleuses produisent des effets homo- et contra-latéraux inverses, l'excitation simultanée des fibres droites et gauches provoque des réactions complexes, mélange ou alternance d'inhibition et de contraction des extenseurs. La complexité de ces réactions ressemble fort à celle des réflexes spinaux. Ce fait suggère que la complexité de ces réflexes est due elle aussi à l'excitation de fibres fonctionnellement différentes ainsi que l'avait supposé SHERRINGToN.

13. Le "rebound" d'extension qui suit les ị̣hibitions montre souvent un phénomène de facilitation portant sur sa latence et sur son amplitude. L'existence de cette facilitation est en faveur de l'hypothèse de BERITOFf de la nature réflexe des phénomènes de rebound. 


\section{BIBLIOGRAPHIE}

1. F.-J. BUYTENDYK. Zeits. . Biol., 1912, CLIX, p. 37.

2. Einthoven. Arch. neerl. Physiol., 1918, II, p. 448.

3. A. FORBES et W.-C. RAPPLEYE. Amer. Journ. of Physiol., 1916, XLII, p. 228.

4. H. SChaeffer: Arch. f. d. ges. Physiol., 1920, CLXXXV, p. 42.

5. Liljestrand et MagnuS. Arch. $f$. d. ges. Physiol., 1919, CLXXVI, p. 168.

6. WertTheim-Salomonson. Brain, 1920, XLII, p. 169.

7. Magnus et De Kleyn. Arch. f. d. ges. Physiol., 1912, CXLV, p. 455.

8. T. Graham Brown. Proc. Roy. Soc., 1913, LXXXVII, B, p. 145.

9. GORDON HOLMES. Brain, 1917, XL, p. 461.

10. De Kleyn et Magnus. Arch. f. d. ges. Physiol., 1920, CLXXVIII, p. 177.

11. Bolk. Das Cerebellum der Säugetiere, Bohn-Haarlem, 1906.

12. Troell et HoSSe r. Acta. Chir. Scand., 1921, LIV, p. 211.

13. S. IngVaR. Folia neurobiologica, 1918, XI, p. 395.

14. THIELE. Journ. of Physiol., 1905, XXXII, p. 359.

15. ShERRINGTON. Journ. of Physiol., 1897, XXII, p. 319.

16. TRETIAKOF F et. BREMER. Revue neurologique, 1920, X X XVII, p. 772.

17. V. HORSLEY. Brain, 1906, XXIX, p. 446.

18. WEED. Journ. of Physiol., 1914, XLVIII, p. 205.

19. K. WILSON. Brain, 1920, XLIII, p. 265.

20. Lefwenthal et Horsley. Proc. Roy. Soc., 1897, LXI.

21. S. CớB, A. Bailey et P. Holtz. Am. J. of Physiol., 1917, XLIV, p. 239.

22. HorSLEY et ClARKE. Brain, 1908, XXXI, p. 44.

23. Mille et SherRington. Quart. Journ. Exper. Physiol., 1915, IX, p. 147.

24. Goltz. Arch. f. d. ges. Physiol., 1892, LI, p. 570.

25. DUSSER et BURENne. Arch. neerl. Physiol., 1919, IV, p. 31.

26. R. BING. a) Die Bedeutung der spino-cerebellaren Systeme. Wiesbaden, 1907 ;

b) Arch. f. Anal. u. Physiol. Physiol. Abt., 1906, p. 250.

27. MAC NALTY et HORSLEY. Brain, 1909, XXXII, p. 236.

28. Clarke et Horsley. Brain, 1905, XXVIII, p. 13.

29. ANDRÉ-ThOMAS. Le cervelet, étude anatomique, clinique et physiologique Paris, 1897.

30. Van GehuChten. Anatomie du système nerveux. Louvain, 1906.

31. A. Froelich et Sherrington. Journ. of Physiol., 1902, XXVIII, p. 14.

32. SherRIngton et LASLeTt. Journ. of. Physiol., 1903, X XIX, p. 188.

33. ANDRÉ-Thomas. La fonction cérébelleuse. O. Doin, Paris, 1911, p. 107.

34. W. Trendelenburg. Arch. f. Physiol., 1908, p. 120.

35. Bauer et LeIdler. Arch. Neurol. Inst. Vienne, 1911, XVII, p. 153.

36. R. Magnus. Arch. f.d. ges. Physiol., 1916, CLXIII, p. 490.

37. WALSH. Brain, 1921, XLIV, p. 538.

38. Sherrington. Proc. Roy. Soc., 1913, LXXXXI, B, p. 233.

39. A. For BES. Quart. Journ. Exp. Physiol., 1912,.V., p. 149.

40. Sherrington. Quart. Journ. Exp. Physiol., 1913, VI, p. 252.

41. Forbes. Amer. Journ. of Physiol., 1921, LVI, p. 273.

42. BERITOFF. Quart. Journ. of Exp. Physiol., 1915, VIII, p. 198.

43. Sherrington. Quart. Journ. of Exp. Physiol., 1909, II, p. 109. 